\title{
REVIEW
}

\section{On-chip light sources for silicon photonics}

\author{
Zhiping Zhou ${ }^{1}$, Bing Yin ${ }^{1}$ and Jurgen Michel $^{2}$
}

Serving as the electrical to optical converter, the on-chip silicon light source is an indispensable component of silicon photonic technologies and has long been pursued. Here, we briefly review the history and recent progress of a few promising contenders for on-chip light sources in terms of operating wavelength, pump condition, power consumption, and fabrication process. Additionally, the performance of each contender is also assessed with respect to thermal stability, which is a crucial parameter to consider in complex optoelectronic integrated circuits (OEICs) and optical interconnections. Currently, III-V-based silicon (Si) lasers formed via bonding techniques demonstrate the best performance and display the best opportunity for commercial usage in the near future. However, in the long term, direct hetero-epitaxial growth of III-V materials on Si seems more promising for low-cost, high-yield fabrication. The demonstration of high-performance quantum dot (QD) lasers monolithically grown on Si strongly forecasts its feasibility and enormous potential for on-chip lasers. The superior temperature-insensitive characteristics of the QD laser promote this design in large-scale high-density OEICs. The Germanium (Ge)-on-Si laser is also competitive for large-scale monolithic integration in the future. Compared with a III-V-based Si laser, the biggest potential advantage of a Ge-on-Si laser lies in its material and processing compatibility with Si technology. Additionally, the versatility of Ge facilitates photon emission, modulation, and detection simultaneously with a simple process complexity and low cost.

Light: Science \& Applications (2015) 4, e358; doi:10.1038/lsa.2015.131; published online 20 November 2015

Keywords: on-chip light sources; optical interconnections; optoelectronic integrated circuit; silicon photonics

\section{INTRODUCTION}

With sustained exponential performance growth in the information industry, global Internet traffic is set to grow dramatically and will surpass the zettabyte ( $2^{70}$ bytes) threshold in $2016^{1}$; because of this trend, broadband, high-density, high-speed data interconnections at a low-cost are strongly desired for signal processing and high-performance computing. Optical interconnections via silicon (Si) photonic technology have been widely recognized as a potential solution to overcome this bottleneck. Higher data rates and higher interconnection densities with lower system-level power dissipations could be obtained when using photons as the medium to detect, process, and transmit information ${ }^{2}$. Over the past two decades, an unprecedented technology boom has occurred in silicon photonics. A few keystone components have been demonstrated with superior performance, such as the extremely low-loss waveguide ${ }^{3}$, ultra-fast modulator ${ }^{4}$, and high bandwidth detector ${ }^{5}$. Additionally, the progress achieved in the field of photonic integration is also exciting, with the successful demonstration of a high-density optical interconnect system with over 30 -Tbps $\mathrm{cm}^{-2}$ bandwidth density ${ }^{6}$ and a monolithic integrated Ge-based photonic interconnection ${ }^{7}$.

Recently, the respective merits and flaws of on-chip versus off-chip light sources for optical interconnections have been comprehensively analyzed and discussed ${ }^{8}$. An off-chip light source displays high lightemitting efficiency and good temperature stability but suffers from relatively large coupling losses between the off-chip light source and the Si chip and a high packaging expense. On the contrary, an on-chip light source could potentially achieve a higher integration density with a compact size and display a better performance in terms of energy efficiency and energy proportionality. Despite these substantial advantages, the development of an on-chip light source on Si has seriously lagged behind that of other photonic components mentioned above $\mathrm{e}^{3-5}$ because of the low emission efficiency of $\mathrm{Si}^{9}$, currently limiting the progress of optical interconnection technology. A few promising candidates have been extensively researched, including porous $\mathrm{Si}^{10}, \mathrm{Si}$ nanocrystals ${ }^{11}$, Si Raman lasers ${ }^{12}$, Erbium (Er)-related light sources $^{13-16}$, Germanium (Ge)-on-Si lasers ${ }^{17-20}$, and III-V-based Si lasers $^{21-24}$. To fully utilize the current microelectronics and optical communication technologies, an ideal light source should satisfy the following requirements ${ }^{17}$ : (1) emit at approximately 1310 or $1550 \mathrm{~nm}$ to connect directly with the fiber optical network; (2) lase under electrical pumping for a compact size and high integration density; (3) display high power efficiency for sufficient output power and low energy cost-per-bit in data transmission; and (4) integrate on Si with complementary metal oxide semiconductor (CMOS)-compatible fabrication techniques for large-scale manufacturing. Accordingly, porous Si, Si nanocrystals, and Si Raman lasers fail to meet the aforementioned requirements because of wavelength mismatch, ultralow emission efficiency, or intrinsic optical-pumping mechanisms. Given their comprehensive performance, current studies primarily focus on the following three types of promising light sources: (1) Er-related light source, (2) Ge-on-Si laser, and (3) III-V-based Si laser. Table 1 summarizes the basic information of these three candidates. Each candidate

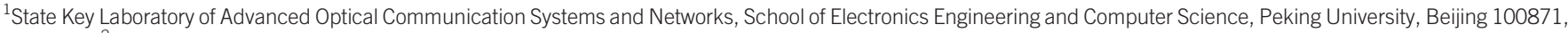

China and ${ }^{2}$ Department of Materials Science and Engineering, Massachusetts Institute of Technology, Cambridge, MA 02139, USA

Correspondence: Z Zhou, Email: zjzhou@pku.edu.cn

Received 25 December 2014; revised 4 July 2015; accepted 4 August 2015; accepted article preview online 5 August 2015 
Table 1 Key strengths and challenges of each promising candidate

\begin{tabular}{|c|c|c|c|}
\hline Candidates & Er-related light source & Ge-on-Si laser & III-V-based Si laser \\
\hline Working mechanism & $\begin{array}{l}\text { Using Er as an atomic emitting } \\
\text { center }\end{array}$ & $\begin{array}{l}\text { Enhance emitting efficiency via bandgap } \\
\text { engineering }\end{array}$ & Using III-V material as gain medium \\
\hline Gain material & $\mathrm{SiO}_{x}: \mathrm{Er}^{14} ; \mathrm{SiN}_{x}: \mathrm{Er}^{15} ;$ Er silicates $^{16}$ & $\mathrm{Ge}^{17-19} ; \mathrm{GeSn}$ alloy ${ }^{20}$ & InAlGaAs QW ${ }^{21,22}$; InGaAsP QW ${ }^{23} ;$ InAs/GaAs QD 24 \\
\hline Advantage & $\begin{array}{l}\text { CMOS compatible fabrication } \\
\text { process; Wavelength stability }\end{array}$ & $\begin{array}{l}\text { Large gain spectrum; Material and process } \\
\text { compatibility with Si technology }\end{array}$ & $\begin{array}{l}\text { High gain and output optical power; Good structure } \\
\text { design flexibility }\end{array}$ \\
\hline Challenge & Low EL efficiency; Obtain net gain & Ge materials quality; Ultrahigh threshold current & Fabrication compatibility and cost reduction \\
\hline
\end{tabular}

EL stands for electroluminescence; QW and QD stand for quantum well and quantum dot, respectively.

displays unique properties and advantages. In this paper, therefore, a comprehensive analysis has been conducted and includes a review of the history, a summary of recent progress, and an assessment of the development trends from the perspective of realizing an ideal on-chip light source for large-scale optoelectronic integrated circuits (OEICs).

\section{ER-RELATED LIGHT SOURCE}

The ability to emit at approximately $1.55 \mu \mathrm{m}$ via its unique intra- $4 \mathrm{f}$ transition and the extraordinary success of Er-doped fiber amplifiers and lasers (EDFAs/EDFLs) have greatly stimulated the development of an Er-related laser on Si. However, compared with EDFAs/EDFLs, the smaller size and electrical pumping requirements of Er-related lasers introduce additional difficulties in terms of fabrication, material gain coefficients, and energy transfer efficiencies. For example, optically pumped Er-related lasers ${ }^{25,26}$ were successfully demonstrated a decade ago, whereas no electrically pumped Er-related laser has ever been reported. According to the conduction mechanism, the fully explored electrical pumping Er-related light sources can be divided into two basic groups: (1) Er-doped Si-rich materials and (2) Er compound materials. The first group of materials obeys the rule of the PooleFrenkel conduction mechanism ${ }^{27}$ and mainly includes Er-doped Si-rich Si dioxide $\left(\mathrm{SiO}_{\mathrm{x}}: \mathrm{Er}\right)^{28}$ and Er-doped Si-rich $\mathrm{Si}$ nitride $\left(\mathrm{SiN}_{\mathrm{x}}: \mathrm{Er}\right)^{29}$. The second group of materials follows the rule of the Fowler-Nordheim (F-N) tunneling mechanism ${ }^{30}$ and includes erbium silicates ${ }^{31}$ and erbium chloride silicate (ECS) ${ }^{32}$.

In Er-doped Si-rich materials, the excess $\mathrm{Si}$ is in the form of $\mathrm{Si}$ nanocluster (Si-nc). The typical energy level scheme for the coupled Er:Si-nc system ${ }^{28}$ is described in Figure 1. The Si-nc acts as a sensitizer that transfers the absorbed energy to Er ions, quantified by the transfer coefficient $\mathrm{C}_{\mathrm{nc} \text {-Er }}$. Additionally, the Si-nc utilizes quantum effects to localize the carriers and provides a spatial constriction of electronhole pairs. Therefore, the probability of radiative recombination is increased, thus enhancing the internal quantum efficiency (IQE) of the material. However, the introduction of excess $\mathrm{Si}$ also results in

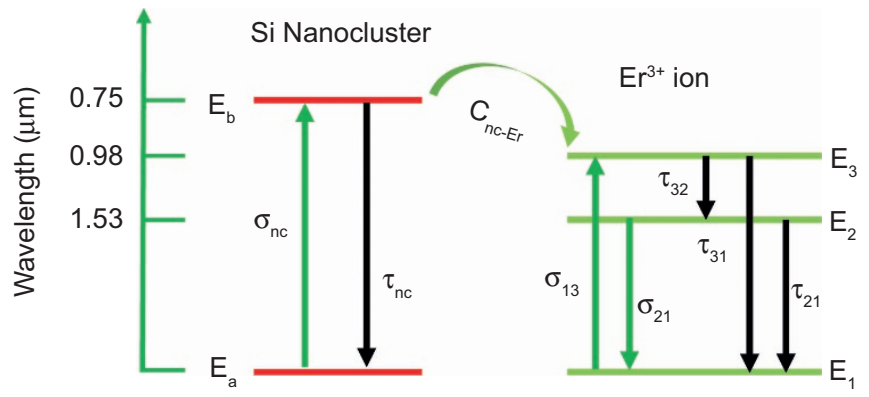

Figure 1 Energy levels of Er and silicon Si-nc in Er-doped Si-rich materials. $\mathrm{Si}-\mathrm{ncs}$ and $\mathrm{Er}^{3+}$ are modeled as a two and three level system, respectively. $\mathrm{C}_{\mathrm{nc}-\mathrm{Er}}$ is the transfer coefficient between Si-ncs and $\mathrm{Er}^{3+}$. This figure is reproduced from Ref. 28, (c) 2010 American Institute of Physics. nonradiative processes such as free carrier absorption (FCA), in which free carriers in the conduction band absorb photons and jump to higher energy levels. The enhancement of emission efficiency is therefore severely limited. Additionally, the energy back-transfer from erbium to Si exists simultaneously, thus limiting the energy transfer efficiency and the obtainable concentration of excited erbium ions.

Therefore, means to accomplish unidirectional energy transfer and to suppress FCA effectively are still urgent issues that must be addressed. The effects of annealing treatment ${ }^{33}$ and polarization strategy $^{34}$ on the device performance have been analyzed and optimized. Additionally, a novel horizontal multi-slot structure was proposed ${ }^{35}$ to enhance the emission efficiency, in which the energy back-transfer and FCA were suppressed under lateral carrier injection because of unique photon confinement ${ }^{36}$. Another method to improve light source performance is the introduction of a surface plasmon (SP) into the light-emitting device because the SP could increase the spontaneous emission rate and $\mathrm{IQE}^{37,38}$. Unfortunately, the enhanced wavelength is only effective in the visible region. Despite these improvements, the effect is limited. In addition, Er-doped materials suffer from a low erbium concentration because of the finite erbium solubility in $\mathrm{Si}$. Further increases in erbium concentration, such as in heavy doping, lead to a nonuniform distribution and erbium ions clustering $^{39}$. Experimental results indicated that this increased concentration significantly reduced the population of optically active erbium ions ${ }^{40}$, complicating the realization of an adequate gain in waveguides within sub-millimeter length scales. Therefore, no net optical gain in the Er-doped Si-rich materials has yet been reported under electrical pumping. The electrically excited $\mathrm{Er}^{3+}$ ions achieved were only up to $20 \%$ in experiment with a power efficiency of $10^{-2} \%{ }^{41}$.

To further increase the erbium concentration, erbium compound materials including erbium silicate and ECS have become an active topic of research because of their stoichiometric nature. Erbium silicate is the first widely researched erbium compound material in which almost all of the erbium ions are optically active ${ }^{16}$. Benefiting from the high erbium concentration, a strong infrared photoluminescence (PL) at room temperature (RT) has been experimentally observed in erbium silicate ${ }^{42}$. Nevertheless, the high erbium concentration shortens the distance of neighboring erbium ions, resulting in strong cooperative upconversion $(\mathrm{CU})$ and concentration quenching, which are detrimental factors for achieving material gain. An effective strategy to address this issue is introducing ytterbium $(\mathrm{Yb})$ and yttrium $(\mathrm{Y})$ ions into the erbium silicates to dilute the erbium ions because they share a similar ionic radius (Y: $0.90 \AA$, Er: $0.88 \AA$, Yb: 0.86 $\AA$ ) and silicate crystal structure. Consequently, the CU and concentration quenching were suppressed, and the infrared PL efficiency was greatly enhanced by optimizing the composition of $\mathrm{Yb}$ and $\mathrm{Y}$ additions ${ }^{43-45}$. Optical amplification was comprehensively analyzed and measured in $\mathrm{Er} / \mathrm{Yb} / \mathrm{Y}$ silicate waveguides ${ }^{46,47}$, and a $30 \mathrm{~dB} \mathrm{~cm}^{-1}$ modal gain was demonstrated in a Si photonic crystal slot structure ${ }^{47}$. In addition, electroluminescence (EL) was observed in experiments 
via hot-electrons impact excitation, and recent studies suggested that a strong potential material gain of more than $23 \mathrm{~dB} \mathrm{~cm}^{-1}$ in $\mathrm{Er} / \mathrm{Yb}$ silicate may be achieved under low current density excitation ${ }^{48}$.

ECS was first synthesized in 2011 in the form of single crystal nanowires $^{32}$ with a long lifetime and a high erbium concentration simultaneously. The highest erbium concentration achieved was $1.6 \times 10^{22} \mathrm{~cm}^{-3}$, and the lifetime can be as long as $540 \mu \mathrm{s}^{49}$. To assess the suitability of various Er-materials for light emission, the lifetimedensity product (LDP) was proposed as a convenient figure-of-merit quantity and ECS has the highest LDP value (Figure 2a). Moreover, the gain simulation analysis indicated that ECS can achieve enormous optical gains under low excitation photon fluxes (Figure 2b). This result was supported by the successful demonstration of an ECS nanowire waveguide with at least $30 \mathrm{~dB} \mathrm{~cm}^{-1}$ net gain ${ }^{50}$. However, similar to erbium silicate, ECS also suffers from the CU effect and concentration quenching. Therefore, $\mathrm{Y}$ was introduced into ECS to control and optimize the erbium concentration, and an optical gain exceeding $100 \mathrm{~dB} \mathrm{~cm}^{-1}$ is currently achievable under optical pumping in optimized erbium yttrium chloride silicate ${ }^{51}$.

The main problem with erbium compound materials is their dielectric characteristics, hindering the transmission of electrical currents. Although EL has been clearly observed in ErYb/Y silicates via hot-electron impact excitation, a high electrical field is required to accelerate the carriers to become hot, thus rendering a high threshold voltage. For erbium yttrium chloride silicate materials, no EL has yet been experimentally demonstrated. Moreover, the incompatible CMOS fabrication process also limits the potential for large-scale production.

In conclusion, Er-related light sources, either based on Er-doped materials or compound materials, are potential candidates for an onchip laser, whereas the low power efficiency makes them currently impracticable for on-chip application. Compared with light sources for interband transition, the Er-related light source utilizes erbium as an atomic luminescent center and requires an extra energy transfer process for the erbium ion excitation under an electric pump condition. Hence, the EL mechanism of Er-related light sources is inherently less efficient because of the limited erbium ion excitation efficiency. Moreover, such an extra process also introduces additional problems. Er-doped silicon-rich materials suffer from the bidirectional characteristic of the energy transfer process and limited erbium concentration because of erbium ion clustering. Therefore, means to increase the sensitization efficiency of the Si-nc and to achieve a uniform distribution of erbium ions remain to be determined. Although the emission efficiency is relatively higher for erbium compound materials, these materials suffer from a poor current injection and potential device instability because of high threshold voltages required to realize EL. Therefore, determining how to achieve efficient current injection in erbium compound materials is an upcoming challenge. Improving the material quality or finding suitable protection material with a high breakdown voltage may provide a solution to enhance the device performance. In conclusion, significant development is required before a desired Er-related laser is available, and further investigations must solve the above problems.

\section{GERMANIUM-ON-SI LASER}

Although Ge and Si are both indirect band gap materials, Ge exhibits a pseudo-direct gap behavior because of the small energy difference $(136 \mathrm{meV})$ between the direct valley $(\Gamma)$ and indirect valleys $(\mathrm{L})^{52}$. This unique band structure causes Ge to operate properly at the optical telecommunication wavelength of approximately $1550 \mathrm{~nm}$ and opens the possibility of achieving efficient light emission by engineering its band structure, which was first theoretically demonstrated in $2007^{17}$. Generally, $n$-type doping ${ }^{53}$, introducing tensile strain ${ }^{54,55}$, or using germanium-tin $(\mathrm{GeSn})$ alloy $^{20}$ are the three most widely used routes for modifying the band structure of Ge and eventually turning Ge into a direct bandgap material.

In the $n$-doping method, the extrinsic electrons offered by $n$-type dopants such as phosphorous "fill up" states in the L valley first according to the Fermi distribution, thus increasing the possibility of electrons in the $L$ valley jumping up into the $\Gamma$ valley via the intervalley scattering process. Furthermore, theoretical calculations predicted that the gain of the Ge material increased with doping concentration and the $0.25 \%$ tensile-strained Ge became a direct bandgap material when the activated $n$-type doping concentration reached $7.6 \times 10^{19} \mathrm{~cm}^{-3} 56$. Ion implantation ${ }^{57}$ and in situ doping ${ }^{58}$ are widely researched to obtain high-level $n$-doping concentrations. Ion implantation is accessible to achieve a high doping level, whereas the Ge samples after implantation usually suffer from significant lattice damage and increasing absorption loss. In situ doping occurs during material growth and its main problem consists in the contradiction between the phosphorous out-diffusion and the high temperature $(\mathrm{HT})$ for high-quality materials growth ${ }^{59}$, thus restricting the achievable doping concentration. To address this situation, a
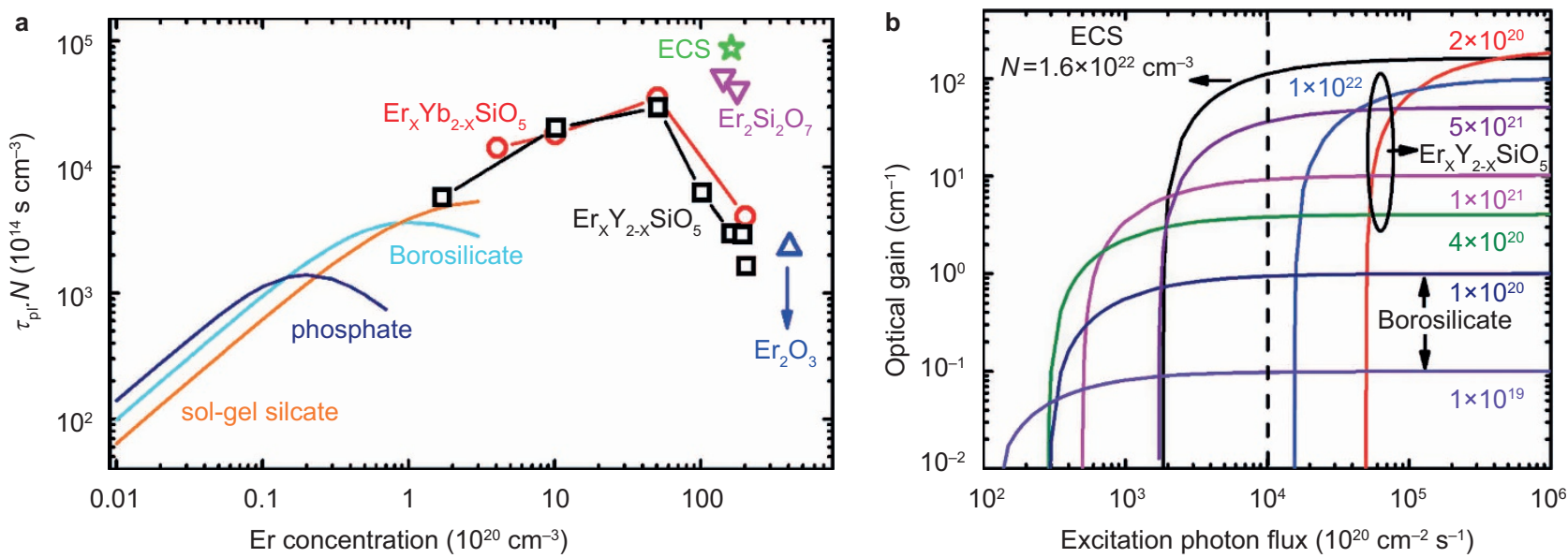

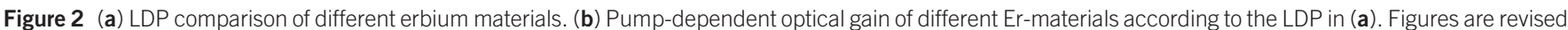
from Ref. 49, (c) 2012 American Institute of Physics. 
multi-layered delta doping method ${ }^{60}$ was proposed by growing $\mathrm{Ge}$ through low temperature deposition and significantly increasing the local doping levels. Consequently, active carrier concentrations above $4 \times 10^{19} \mathrm{~cm}^{-3}$ were achieved by effectively controlling the dopant outdiffusion, representing the best results using a chemical vapor deposition (CVD) growth method ${ }^{61}$.

Tensile strain is another way to tune the Ge bandgap because it causes the Ge bandgap to shrink and the direct bandgap $\left(E_{\mathrm{g}}^{\Gamma}\right)$ reduces faster than the indirect bandgap $\left(E_{\mathrm{g}}^{\mathrm{L}}\right)$, thus decreasing the energy difference. Moreover, the tensile strain also leads to light hole/heavy hole $(\mathrm{LH} / \mathrm{HH})$ splitting, consequently reducing the density of states near the valence band edge and providing an extra benefit of achieving a low-threshold laser. A theoretical analysis indicated that Ge would become a direct band gap material when more than $2 \%$ biaxial tensile strain or $4.6 \%$ uniaxial tensile strain is applied ${ }^{54}$. Several approaches have been extensively explored to accomplish large and flexible tensile strains in Ge. Thermal annealing is the most convenient strategy to introduce biaxial tensile strain because of the thermal expansion coefficient mismatch between $\mathrm{Ge}$ and $\mathrm{Si}^{62}$. Nevertheless, the tensile strain achieved in this process is limited to $0.25 \%$ because of the residual compressive strain in the relaxation at the $\mathrm{HT}^{63}$. Another approach is growing Ge on III-V buffer layers with different lattice parameters such as InGaAs buffer layers ${ }^{64}$ and over $2 \%$ in tensile strain was realized in quantum wells (QWs) and quantum dots (QDs). However, the fabrication process is expensive and not CMOS compatible. Recently, increasing attention has been focused on introducing tensile strain via strain transfer from other intrinsic stressors, notably silicon nitride $\left(\mathrm{Si}_{3} \mathrm{~N}_{4}\right)^{65-67}$, because of its CMOS compatible fabrication and flexibility in realizing either a compressive or tensile stressor by changing deposition parameters. When used as a compressive stressor, $\mathrm{Si}_{3} \mathrm{~N}_{4}$ releases the strain by forcing Ge to expand ${ }^{65}$. When used as a tensile stressor, $\mathrm{Si}_{3} \mathrm{~N}_{4}$ transfers strain to Ge through a contraction on the contact area. The most substantial problem for this method is the low efficiency in the strain transfer process resulting from the large thickness of the substrate, limiting the achievable tensile strain for Ge.

An improved solution was proposed using micromechanical engineering technology for precise structure trimming ${ }^{66,67}$. The efficiency of strain transfer is greatly enhanced by etching through the thick Si substrate or buried oxide layer below the Ge layer to form a suspended platform. Figure 3 a shows the designed structure, in which the Si substrate underlying the $\mathrm{Si}_{3} \mathrm{~N}_{4}$ and Ge-on-insulator (GOI) regions was etched away. Simulation results indicated that large, locally tunable tensile strains could be applied in GOI device layers (Figure 3b). Con sequently, the PL of Ge had been dramatically enhanced over 130 times at $1550 \mathrm{~nm}$ because of the formation of the suspension platform ${ }^{67}$.

Analogous to the tensile strain, adding Sn into Ge also causes the band structure to shrink, and the band gap can be tuned to a wide range by altering the content of $\mathrm{Sn}$ in the GeSn alloy during the growth using CMOS processes. The key for this method is to grow high-quality GeSn alloys on Si with an appropriate Sn content. Currently, the main challenges for this process reside in the large lattice mismatch between $\mathrm{Ge}, \mathrm{Sn}$, and $\mathrm{Si}$ and the low equilibrium solubility $(<1 \%)$ of $\mathrm{Sn}$ in $\mathrm{Ge}^{68}$. Theoretical calculations indicated that GeSn became a direct bandgap with a $6.55 \%$ Sn concentration ${ }^{69}$, whereas higher concentrations of Sn are generally required because of the existence of biaxial compressive strain induced by the large lattice mismatch between Ge and $\alpha$-Sn, complicating the growth of high-quality GeSn alloys. To overcome these obstacles, different buffer layers such as InGaAs and Ge were used, and high-quality GeSn alloys have been successfully grown by molecular beam epitaxy $(\mathrm{MBE})^{70}$ and CVD methods ${ }^{71}$. Moreover, an electrically pumped GeSn laser based on a theoretical design was proposed ${ }^{72}$, and enhanced GeSn direct bandgap luminescence was also demonstrated ${ }^{73}$.

Because of the highly successful application of bandgap engineering technology in Ge, an unexpectedly rapid development of Ge light sources has occurred. Compared with most III-V materials, several unique advantages of germanium have been explored, including the light emission efficiency increasing with temperature ${ }^{74}$ and injection level $^{75}$, the superlinear dependence of the EL intensity on the injection without saturation ${ }^{75}$ and the large gain spectrum ${ }^{56}$. These properties promote Ge light sources as a competitive candidate for Si-compatible on-chip lasers. Moreover, benefitting from introducing $0.25 \%$ tensile strain and $4 \times 10^{19} \mathrm{~cm}^{-3} n$-doping concentration, the first electrically pumped Ge laser ${ }^{19}$ was successfully demonstrated in 2012, as shown in Figure 4a. However, the Ge laser suffered from both a high threshold of $280 \mathrm{kA} \mathrm{cm}^{-2}$ and poor emission efficiency (Figure $4 \mathrm{~b}$ ) restricted by the indirect bandgap structure.

To address this situation, further increase in the $n$-doping concentration is a potential option. However, the $n$-type doping concentration currently achieved is high, and further increases above the solid solubility limit will present difficulties in controlling the dopant outdiffusion and growing high-quality Ge films simultaneously. Furthermore, once the quasi Fermi level is above the $\Gamma$ valley
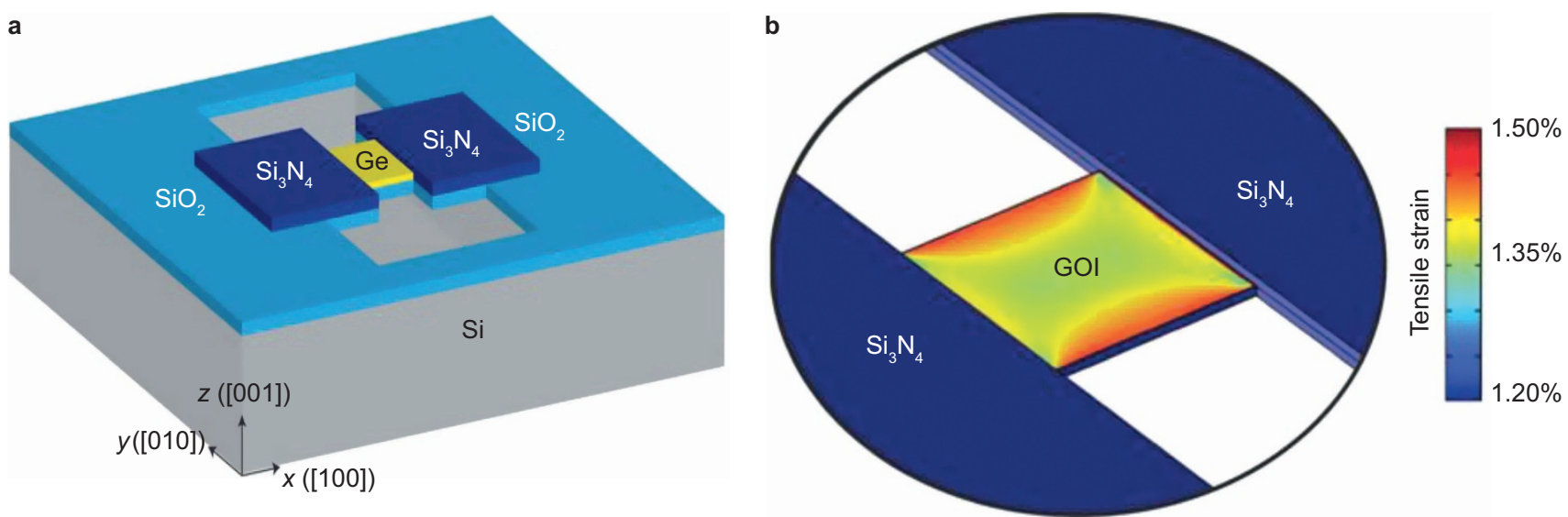

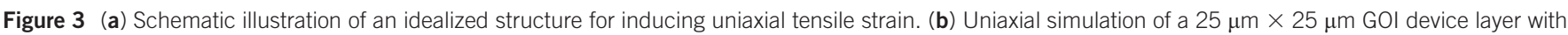

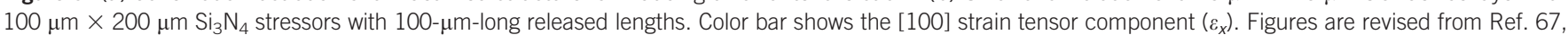
(c) 2012 Macmillan Publishers Limited. 
a

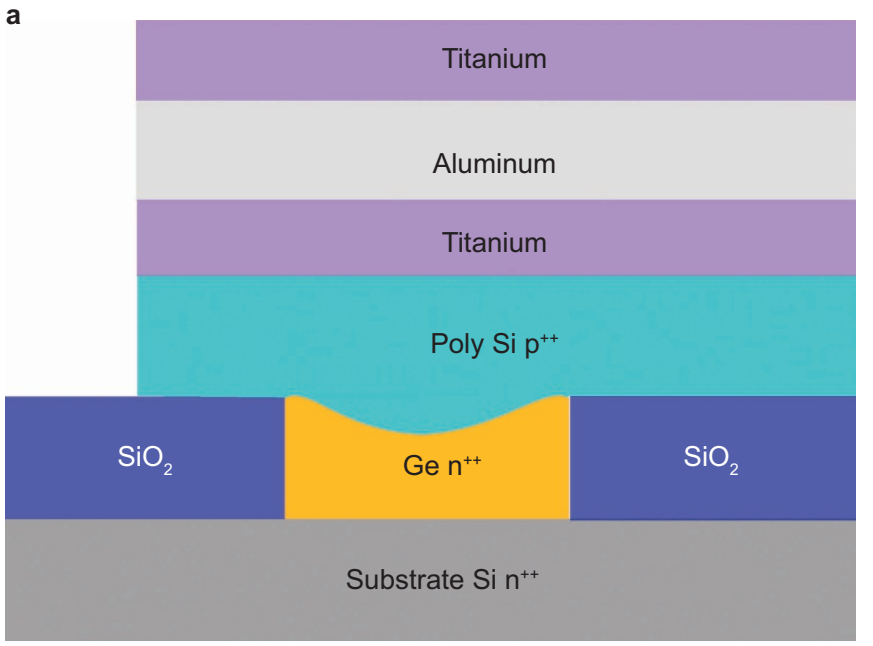

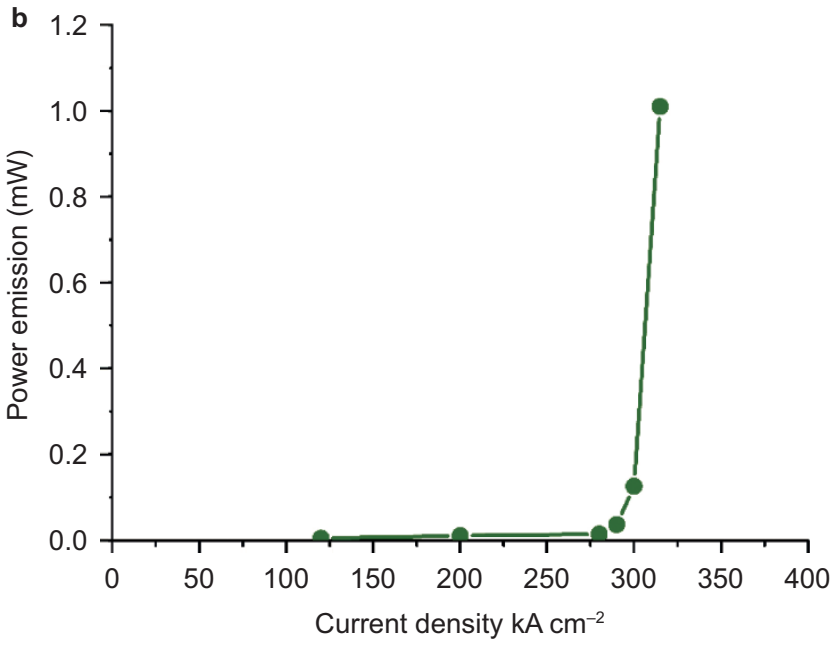

Figure 4 (a) Schematic cross-section of the electrically pumped Ge laser structure; Carrier recombination occurs in the $n++$ Ge region and the width and length of Ge waveguide is 1 and $270 \mu \mathrm{m}$, respectively. The Ge layer thickness is varied between 100 and $300 \mathrm{~nm}$ depending on wafer and location on the wafer. (b) $L-I$ curve of Ge laser measured under electrical pulse condition at $15{ }^{\circ} \mathrm{C}$. Figures are revised from Ref. 19, ๑ 2012 Optical Society of America.

minimum, the HT benefits will be lost. In addition, conflicting results are noted in the literature both in theoretical and experimental studies. On the one hand, although the FCA and Auger recombination are expected to increase rapidly with doping level, simulations have shown that small threshold currents can be achieved even at doping levels up to $10^{20} \mathrm{~cm}^{-3}$, assuming that the minority carrier lifetime is on the order of milliseconds ${ }^{76}$. Experiment result also indicated that the $\mathrm{PL}$ intensity increased with doping concentrations of at least up to $3.5 \times 10^{19} \mathrm{~cm}^{-3} 77$. On the other hand, an experimental study has shown that when the doping concentrations exceeded $3 \times 10^{19} \mathrm{~cm}^{-3}$, both the peak and integrated EL intensity decreased sharply ${ }^{78}$.

Theoretical analyses ${ }^{79}$ have noted that tensile strain is more efficient than $n$-typed doping in terms of threshold reduction and can effectively decrease the required level of $n$-doping for a low threshold Si-based Ge laser. Therefore, increasing the tensile strain is a promising option for improving the Ge laser performance. Attempts to increase this strain have resulted in the design of novel structures, such as nano-array pillars $^{80}$ and microbridges ${ }^{81-82}$. Large tensile strains were realized by reducing the contact area and enhancing the strain transfer efficiency. Over $3.1 \%$ of uniaxial tensile strain was experimentally obtained in a microbridge structure ${ }^{82}$ and that a net gain of $460 \mathrm{~cm}^{-1}$ was theoretically achievable at $0.6 \mathrm{eV}$, as indicated in Figure 5. Furthermore, over $5.0 \%$ of uniaxial tensile strain was realized in the Ge microbridge structure by transferring $\mathrm{Ge}$ on $\mathrm{SiO}_{2}$ via wafer bonding ${ }^{83}$, and $\mathrm{Ge}$ eventually became a direct bandgap material. These results are promising whereas challenges still exist. The increased strain narrows the bandgap and redshifts the emission wavelength from the technologically important $1550-\mathrm{nm}$ band to longer wavelengths. Additionally, lasing of highly strained Ge has not yet been demonstrated.

For the GeSn alloy, a theoretical analysis indicated that it could achieve large optical gains ${ }^{69}$ and be efficient in reducing the lasing threshold of Ge lasers ${ }^{84}$ when compared with $n$-doping. This result has been confirmed in an experimental demonstration of the first optically pumped GeSn laser using CMOS processing ${ }^{85}$. The key challenge for this design lies in growing a high-quality direct gap GeSn layer with Sn contents of up to $12.6 \%$. This challenge is elegantly solved by depositing relaxed GeSn layers onto strainrelaxed Ge buffer layers on silicon, and the strain is efficiently relaxed by the so-call Lomer dislocation ${ }^{86}$. Although such a laser only lased up to $90 \mathrm{~K}$, this design strongly indicated the feasibility of using a GeSn alloy to achieve a Ge-on-Si laser with substantial room for improvement.

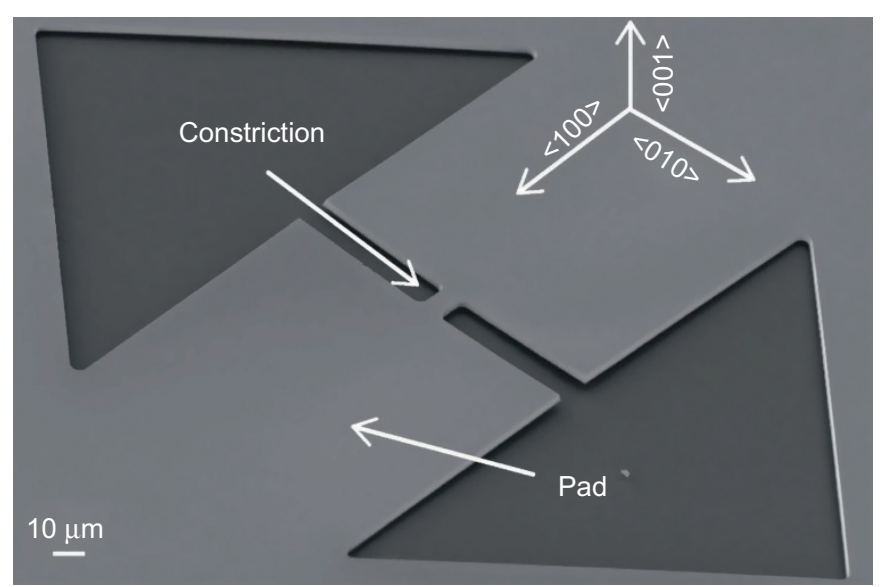

b

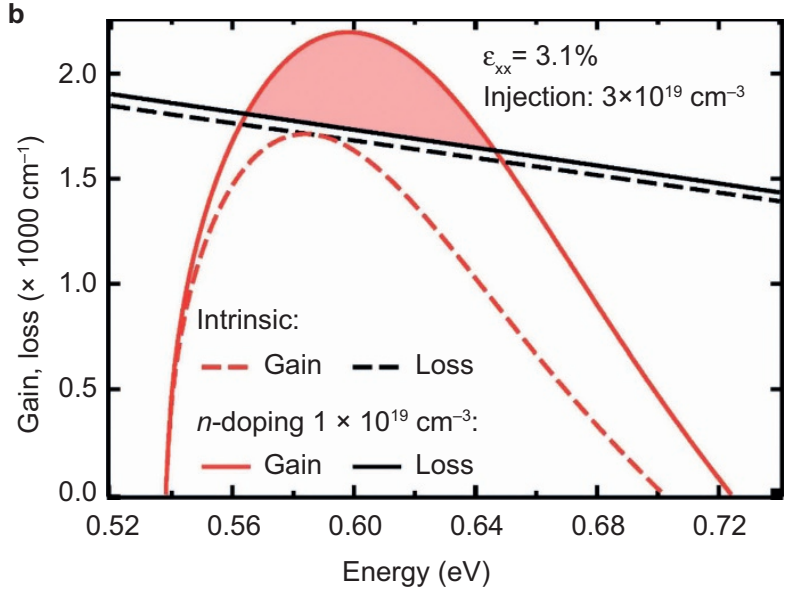

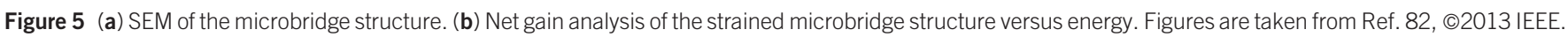


In conclusion, the demonstrations of the electrically pumped $\mathrm{Ge}$ laser and optically pumped GeSn laser are milestone works toward the development of a direct bandgap group IV laser. Additionally, the ability to operate at relative HT and the large gain spectrum increases the attractiveness of the Ge laser in wavelength division multiplexing (WDM) systems and high-density OEICs. In the foreseeable future, the influence of $n$-doping may be reduced, whereas the tensile strain and GeSn alloy could play an increasingly important role in further improving the performance of the Ge laser. Meanwhile, the wavelength red shift caused by these two methods is serious and should also be considered. Hence, an appropriate $n$-doping concentration is still indispensable for mitigating the redshift effects. Additionally, the delicate balance between the aforesaid methods remains a critical issue in the perspective of optimizing the performance of the Ge laser.

\section{III-V-BASED SI LASER}

Benefitting from the direct bandgap structure and high material gain, integrating III-V semiconductors on Si substrate is currently the most practical way of obtaining on-chip light sources in silicon photonics. The main challenge lies in the significant mismatch between the Si and III-V materials in terms of lattice constants and thermal expansion coefficients. This mismatch results in a high density of defects such as threading or misfit dislocation at the interface and large power losses, thus impeding the direct growth of III-V gain materials on a siliconon-insulator (SOI). To address this issue, three main integration approaches have been extensively explored: direct mounting integration, wafer bonding-based heterogeneous integration, and direct hetero-epitaxial growth. Direct mounting integration indicates the direct integration of individual laser diode dies on a SOI wafer via solder bumps, allowing for pre-testing of devices before integration, and potentially maintaining the superior characteristics of the laser chip with good thermal dissipation. The main issue for this method resides in the efficient end coupling between the III-V gain material and the silicon waveguide. Consequently, submicron precision alignment is generally required, which is time consuming and increases the overall assembly cost. Pre-recess patterned trenches ${ }^{87}$ and mode-size converters were explored to ease the alignment requirement in the vertical and horizontal directions. Another issue is the reflection and feedback of light between the output facet of the laser and silicon waveguide, which is detrimental to the overall performance of the laser. Luxtera recently developed a new packaging approach to address this issue utilizing microoptics and placing an optical isolator between them. Ball lenses were used to loosen the alignments restriction. A turning mirror and planar grating were used to accomplish nearvertical coupling. However, the integration density may be limited by

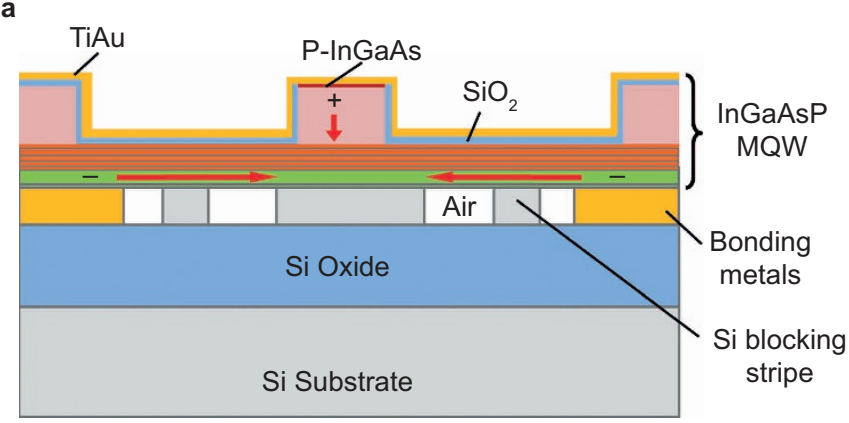

the pitch and size of the solder bumps, and the strenuous fabrication process complicated mass production.

Wafer bonding-based heterogeneous integration involves integrating an unpatterned III-V epitaxial layer on patterned silicon circuits via bonding techniques, and the light generated in the III-V material is vertically coupled into silicon circuits by evanescent coupling. Therefore, this design is not subject to lattice match limitations, providing the possibility of combining the superior gain characteristics of the III-V material and excellent passive characteristics of Si waveguides together. The patterned III-V gain medium and silicon circuits are lithographically aligned with high precision, a key asset of the bonding approach and a crucial advantage when compared to direct mounting integration. According to the stacking technology, bonding techniques include wafer-to-wafer (W2W) bonding and multiple dieto-wafer (D2W) bonding. The former is favorable for massive production, but sensitive to the wafer bowing. Additionally, the large wafer size mismatch between III-V materials (typically 2 inches) and the SOI (typically 8 or 12 inches) increases the difficulty in manufacturing at scale. On the contrary, the latter has a better flexibility and is more suitable in terms of integrating the III-V material into the silicon. Bonding techniques could also be divided into indirect bonding $^{88-92}$ and direct bonding ${ }^{21-24}$ in terms of whether an insertion layer is noted or not. Indirect bonding using metal or polymers such as divinylsiloxane-benzocyclobutane (DVS-BCB or BCB) to act as adhesives and are mainly comprised of metal-assisted adhesive bonding $^{88,89}$ and BCB-assisted adhesive bonding ${ }^{90-93}$.

Metal-assisted adhesive bonding could provide a low resistance Ohmic contact and is effective in thermal dissipation whereas the bonding metal causes a strong light absorption and potential metal contamination. To address these problems, a selective-area metallic bonding technology was demonstrated in which the bonding metal in the light coupling area was removed ${ }^{88}$ (Figure 6a). In addition, an alternative conductive material-indium-tin oxide (ITO)-was also proposed $^{89}$, which is almost transparent in the near infrared (Figure 6b).

$\mathrm{BCB}$-assisted adhesive bonding requires no complicated fabrication and is free from material limitations. $\mathrm{BCB}$ is an appropriate adhesive and exhibits excellent physical properties such as a high bond strength and thermal stability and a low refractive index and curing temperature ${ }^{90}$. Nevertheless, BCB suffers from poor thermal conductivity because of its insulation property, generating a high thermal resistance $\left(\sim 10 \mathrm{~K} \mathrm{~mW}^{-1}\right)^{91}$. Consequently, hybrid lasers via BCB bonding suffer from self-heating effects, rendering the laser performance degradation. Therefore, heat dissipation should be considered when applying BCB bonding. Additionally, the evanescent optical coupling between the top active III-V layer and the bottom SOI waveguides is

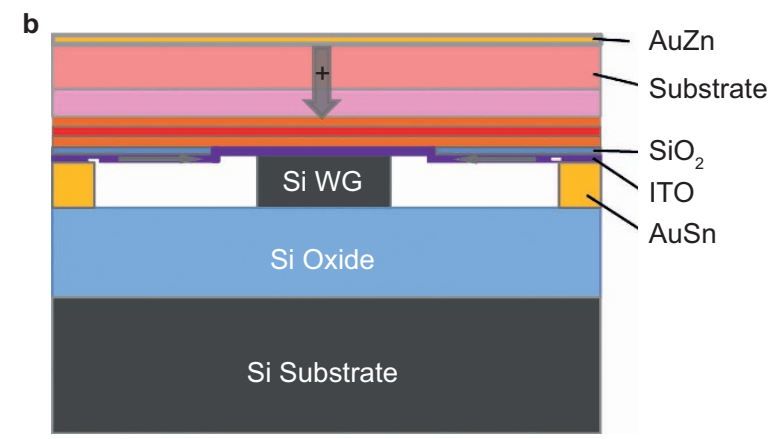

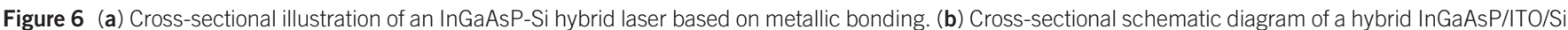
laser. Figures are taken from Refs. 88 and 89, (c) 2010 IEEE and @ 2012 IEEE. 

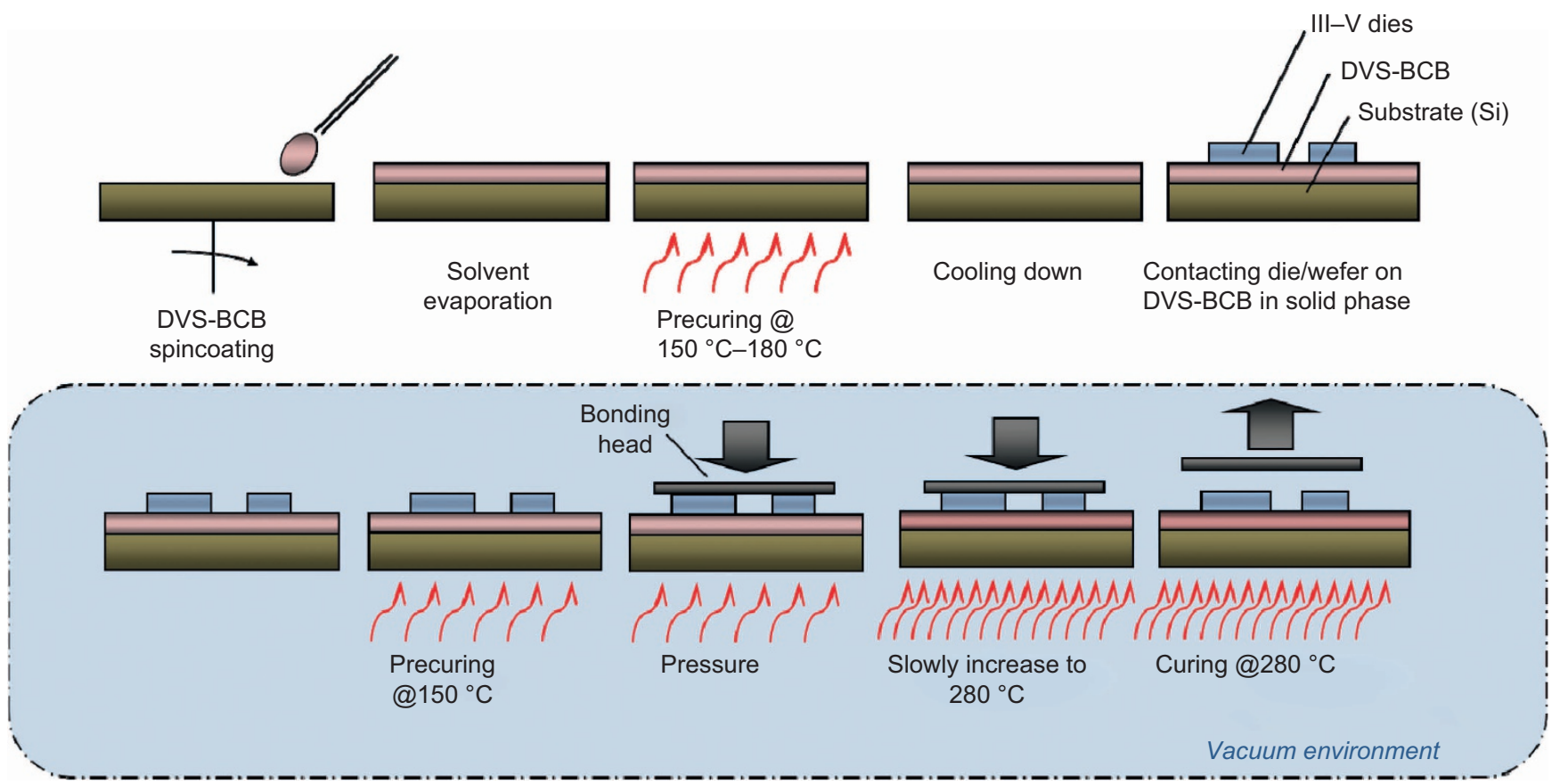

Figure 7 The schematic of developed bonding process, referred to as "cold bonding." This figure is taken from Ref. 92, @ 2012 Optical Society of America.

highly sensitive to the thickness of the intermediate layer between them, e.g. $\mathrm{BCB}$, since the evanescent wave exponential decays in it. Meanwhile, thick BCB layer also restricts the optical confinement in III-V gain material and silicon waveguide, respectively. Determining a technique to achieve a thin BCB layer with a good repeatability became a major challenge in the bonding process. To address this issue, a new "cold bonding" process $^{92}$ was developed (Figure 7 ). Benefiting from this new method, a uniform and ultra-thin bonding layer $(<50 \mathrm{~nm})$ was realized, adequately thin for effective coupling. Accordingly, BCB-assisted adhesive bonding has become a practical approach for the hybrid integration of III-V material on a SOI wafer.
Direct bonding normally refers to a technique that brings mirrorpolished, flat and clean wafers or dies into contact to realize a strong interfacial bond, including the oxygen plasma-assisted low temperature molecular bonding ${ }^{21}$ and hydrophilic molecular bonding ${ }^{93}$. Ultraclean conditions and atom-scale smooth surfaces are generally required to fulfill direct bonding, rendering complex fabrication process. To address this problem, a novel direct fusion bonding technique at low temperatures was proposed with an ideal band gap combination. Based on this technique, a 1.3- $\mu \mathrm{m}$ InAs/GaAs QD laser on Si substrate was demonstrated with low threshold current density of $205 \mathrm{~A} \mathrm{~cm}^{-2} 24$, as illustrated in Figure 8. In addition, the conductive a

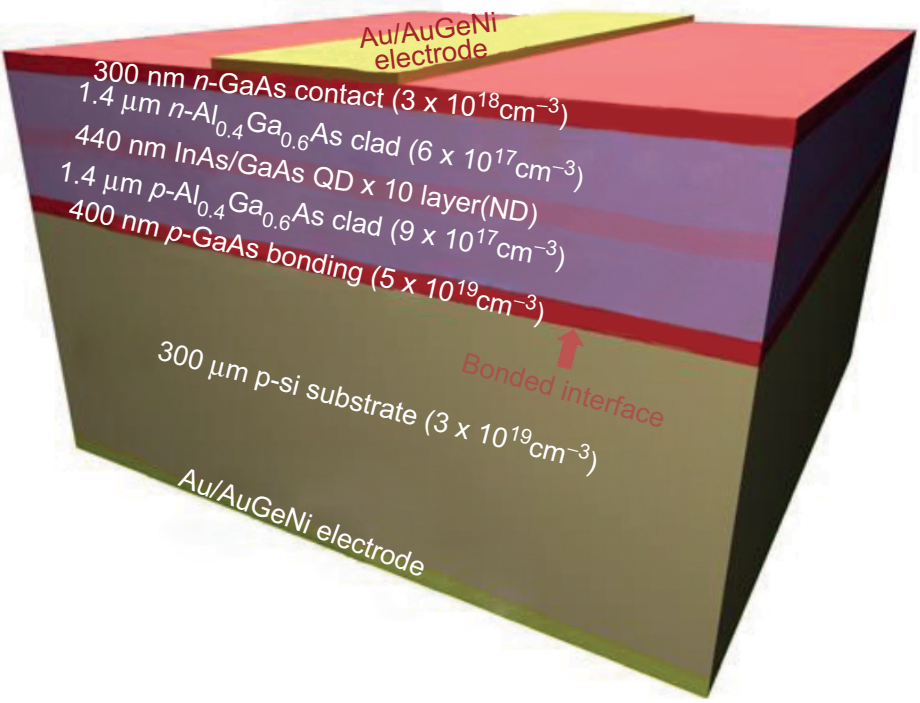

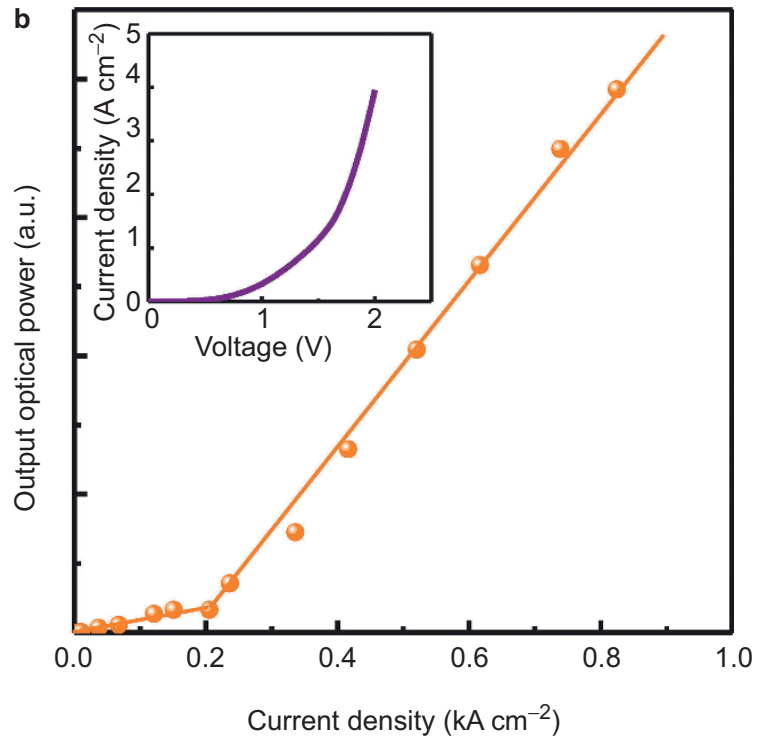

Figure 8 (a) Cross-sectional schematic diagram of the fabricated InAs/GaAs QD laser. (b) $L-I$ characteristics of the laser. The inset shows the $I-V$ characteristics of the laser. Figures are taken from Ref. 24, @ 2012 Macmillan Publishers Limited. 
a

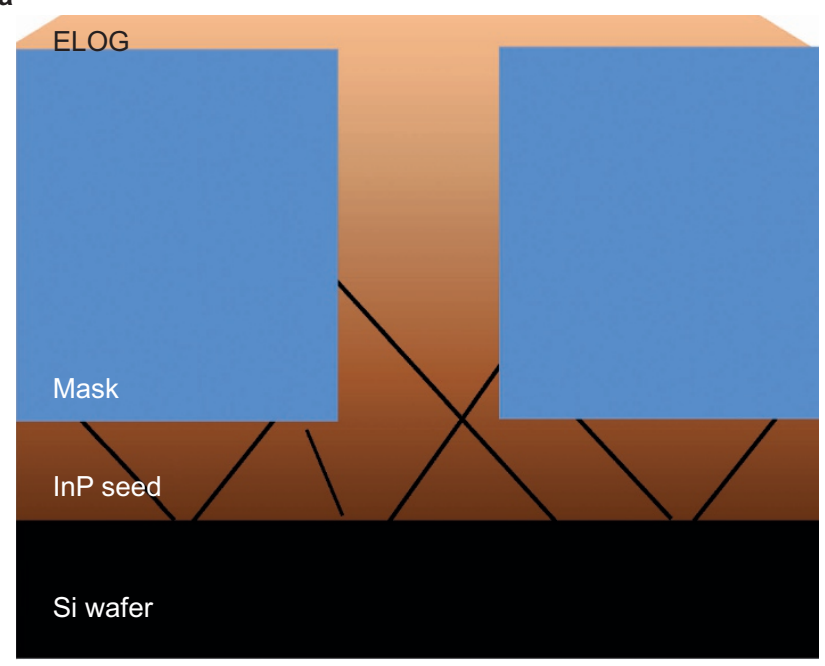

b

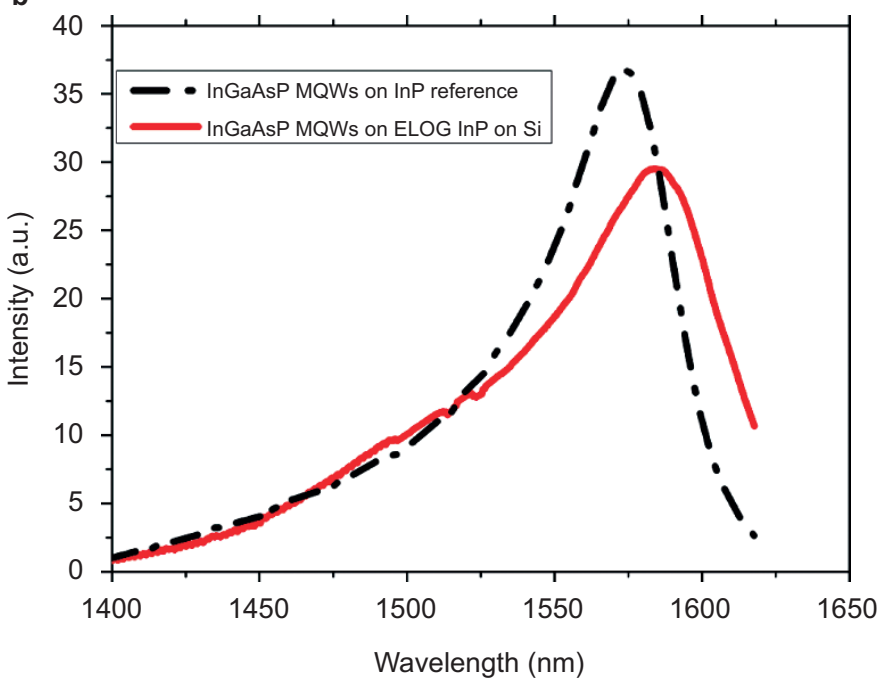

Figure 9 (a) Schematics of epitaxial lateral overgrowth (ELOG) with the defect-necking effect. (b) PL spectra of sample compared with InP reference. Figures are revised from Refs. 101 and 102, (c) 2011 Elsevier B.V. and (C) 2013 IEEE.

wafer-bonded hetero-interfaces enable vertical carrier injection, thus achieving high quantum efficiencies. The potential problems of this technique are the low potential yield during fabrication and lack of reproducibility.

The final integration approach for fabricating III-V-based Si lasers is the direct hetero-epitaxial growth. The main roadblock for this approach resides in the high-density threading dislocations (TDs) resulting from the large difference in thermal and expansion coefficients between Si and III-V compounds. Additionally, the polarity difference between III-V and Si generates antiphase domains at the interface ${ }^{94}$, in which an antisite defect region in the parent forms. Fortunately, this problem could be solved by growing III-V materials on an offcut Si substrate ${ }^{95}$. An efficient method to reduce TDs is inserting a buffer layer between the Si and the III-V active layer with an appropriate lattice constant and similar thermal expansion coefficient. Consequently, $\mathrm{SiGe}^{96}, \mathrm{GaAs}^{97}$, and $\mathrm{GaSb}^{98}$ are widely used and minimize TDs effectively. Furthermore, a new strategic approach to monolithically grow InP on Si with low TDs was proposed based on an epitaxial lateral overgrowth (ELOG) technique ${ }^{99-101}$. The TDs were effectively filtered out by maintaining a high-aspect-ratio between the opening size and mask thickness resulting from the defect-necking effect $^{101}$, which happens when epitaxial growth of materials restrict in a high-aspect-ratio structure. Crystallography dictates that TDs propagate in a nonvertical direction to the surface of the wafer and terminate in the sidewall interface. Therefore, a defect-free top surface layer could be achieved (Figure 9a). Benefitting from the ELOG technique, high-quality QWs successfully grown on the ELOG InP layers were realized showing comparable light emission intensities $(>85 \%)$ with those on a planar InP reference ${ }^{102}$ (Figure 9b).

Another method to suppress TDs is using nano-structures, notably QDs ${ }^{103-110}$, because of their better defect-handling properties. III-V QDs are appealing for temperature-insensitive operations because of a delta-function-like density of states. Additionally, the discrete distribution of QDs also improves the tolerance to defects and offers the capability of filtering TDs, distinguishing them from traditional III-V/ Si QW technologies. A substantial technology boom occurred for QD lasers, notably for InAs/GaAs QD lasers. Electrically pumped $1.3-\mu \mathrm{m}$ InAs/GaAs QD lasers monolithically grown on $\mathrm{Ge}^{105}, \mathrm{Ge}-\mathrm{on}-\mathrm{Si}^{106}$, and $\mathrm{Si}^{107-111}$ substrates have been successfully realized in recent years, as summarized in Table 2. The performance of QD lasers has been significantly improved because of the efficient reduction in the density of TDs. Substantial efforts have addressed this advance, and a new AlAs nucleation layer (NL) has been developed to replace a GaAs NL because of the better defects confinement ability ${ }^{108}$, and this NL layer was experimentally shown to be effective in reducing TDs. Consequently, the QD laser performance was enhanced at a lower threshold current density and higher operation temperature. Moreover, the effects of different filter layers to the performance of a QD laser have also been experimentally explored. Compared to the InGaAs/GaAs strained-layer superlattices (SLSs), InAlAs/GaAs SLSs are more effective in blocking the propagation of TDs and thus rendering a larger PL from InAs/GaAs QDs ${ }^{109}$. Currently, an InAs/GaAs QD laser has shown superior advantages in achieving a large output optical power $^{110}(101 \mathrm{~mW})$ and a high lasing operation temperature ${ }^{111}$

Table 2 Representative performance of InAs/GaAs QD lasers on silicon demonstrated in recent years

\begin{tabular}{|c|c|c|c|c|c|c|c|}
\hline Year & $\lambda(\mu \mathrm{m})$ & Substrate & Operation condition & $J_{\text {th }}\left(A \mathrm{~cm}^{-2}\right)$ & $P_{\text {out }}(\mathrm{mW}) /$ facet & $T_{\max }\left({ }^{\circ} \mathrm{C}\right)$ & Refs. \\
\hline 2011 & 1.302 & $\mathrm{Si}$ & Pulse @RT & 725 & 26 & 42 & 107 \\
\hline 2011 & 1.305 & $\mathrm{Ge}$ & CW@RT & 55.2 & 28 & 60 & 105 \\
\hline 2012 & 1.28 & Ge-on-Si & CW@RT & 163 & 3.7 & 30 & 106 \\
\hline 2014 & 1.27 & $\mathrm{Si}$ & Pulse@20 & 194 & 77 & 85 & 109 \\
\hline 2014 & 1.29 & $\mathrm{Si}$ & Pulse @RT & 200 & 101 & 111 & 110 \\
\hline 2014 & 1.3 & $\mathrm{Si}$ & CW@RT & 141.6 & 88 & 119 & 111 \\
\hline
\end{tabular}

$\mathrm{CW}, J_{\text {th }}, P_{\text {out }}$, and $T_{\max }$ represent continuous-wave, threshold current density, laser output power, and the maximum lasing temperature, respectively. 
$\left(119{ }^{\circ} \mathrm{C}\right)$, indicating the feasibility of the direct hetero-epitaxial growth approach for achieving a monolithic integrated on-chip laser.

With the rapid development of III-V-based hybrid Si lasers, laser power consumption has garnered increasing interest, as it is becoming a significant proportion of the energy cost-per-bit in data transmission. A common parameter to present laser power efficiency is the wall-plug-efficiencies (WPE) defined as the output optical power divided by the electrical input power: $\mathrm{WPE}=P_{\text {out }} /\left(I_{\text {bias }} \cdot V_{\text {bias }}\right)$. Additionally, the adequate output optical power is also indispensable for lasers given the practical application in OEICs. Hence, techniques to enhance the performance of III-V-based hybrid Si lasers in terms of WPE and output optical power have become a focus of much research. One effective approach is to design novel resonant cavities, and III-Vbased hybrid Si lasers in numerous cavities have been achieved including a Fabry-Perot ${ }^{21-23}$, distributed Bragg reflector $(\mathrm{DBR})^{112}$, distributed feedback $(\mathrm{DFB})^{113}$, microring ${ }^{114}$, and microdisk ${ }^{115}$. The experimental results indicated that microrings and microdisks were able to achieve lower threshold hybrid lasers because of their compact size and high quality factor, whereas DFB and DBR were more efficient in accomplishing hybrid lasers with large WPE and output power. Another approach is to reduce the optical coupling loss by realizing the efficient optical coupling between the III-V active layer and the SOI waveguide circuit. Consequently, inverted adiabatic tapers ${ }^{116,117}$ and spot size converters (SSCs) ${ }^{118,119}$ are widely explored and used in evanescently coupled hybrid lasers and butt-coupled hybrid lasers, respectively. A double adiabatic taper coupler structure ${ }^{117}$ was proposed for the efficient evanescent coupling, and over $90 \%$ in coupling efficiency can be obtained in a taper length less than $100 \mu \mathrm{m}$ (Figure 10). For the butt-coupled laser, a novel SSC with a trident Si waveguide (Figure 11a) was demonstrated using a simple fabrication procedure $^{119}$. The coupling loss was as low as $2.3 \mathrm{~dB}$, and the alignment error tolerance was greatly improved in the both horizontal and vertical directions (Figure 11b), effectively enhancing the application of the passive alignment technique for direct mounting integration.

Table 3 compares the key performance of the III-V-based Si laser achieved by the three integration approaches. The III-V-based hybrid Si lasers achieved via direct hetero-epitaxial growth show superior performance in terms of output optical power and working temperature. Compared with evanescently coupled hybrid lasers via heterogeneous integration, butt-coupled hybrid lasers achieved by direct mounting integration show a great advantage in terms of WPE. The WPE of the former at telecom wavelength is still at the level of a few percent while the latter has recently reached around 20\% WPE 6 . One reason for this better performance is that in the evanescently coupled laser, the optical mode is strongly confined in the Si waveguide, thus rendering low optical mode overlaps in the gain media. Therefore, the modal gain and output coupling efficiency are in competition with each other, and the trade-off between them restricts the laser performance. Additionally, for the evanescently coupled laser, the light passes through the heavy doping conducting layer and the bonding layer for vertical coupling, rendering limited carrier injection efficiency and additional absorption loss. Moreover, the evanescently coupled laser performance is also inevitably degraded by characteristics of the bonding layer such as the surface roughness, particle contaminants, and poor thermal conductivity. On the contrary, the butt-coupled laser fabricated by direct mounting could maintain the performance of a laser chip to the maximum extent.

In addition to enhancing the performance of III-V-based hybrid Si lasers, monolithic integration with other functional devices is also one of the ultimate goals. A step forward occurred in 2011 through the demonstration of a Si chip integrating the hybrid Si laser and a compact optical grating with $2.3 \mathrm{~mW}$ power output at $1550 \mathrm{~nm}^{122}$. The next breakthrough was soon reported by Intel through the demonstration of the first 50 Gbps end-to-end silicon photonics connection with four identical hybrid Si lasers and optical modulators integrated together on the identical transmitter ${ }^{123}$. Additionally, multiple bandgap integration was demonstrated using QW intermixing technology via ion implantation enhanced disordering ${ }^{124}$, and consequently, a broadband DFB laser array integrated with electro-absorption modulators was realized on a single chip. A rapid development in this field then occurred, and high-density integrated transmitters and systems were achieved. Among the designs, the world-record highest bandwidth density of over 30 -Tbps $\mathrm{cm}^{-2}$ was achieved in a compact footprint Si optical interposer by integrating hybrid lasers, optical splitters, modulators, waveguides, and photodetectors on a single silicon substrate $^{6}$. Moreover, 20 -Gbps error-free data transmission was also guaranteed simultaneously.

In conclusion, direct mounting integration could achieve a WPE whereas submicron precision alignment will lead to a high assembly cost and limited integration density. Bonding techniques, notably
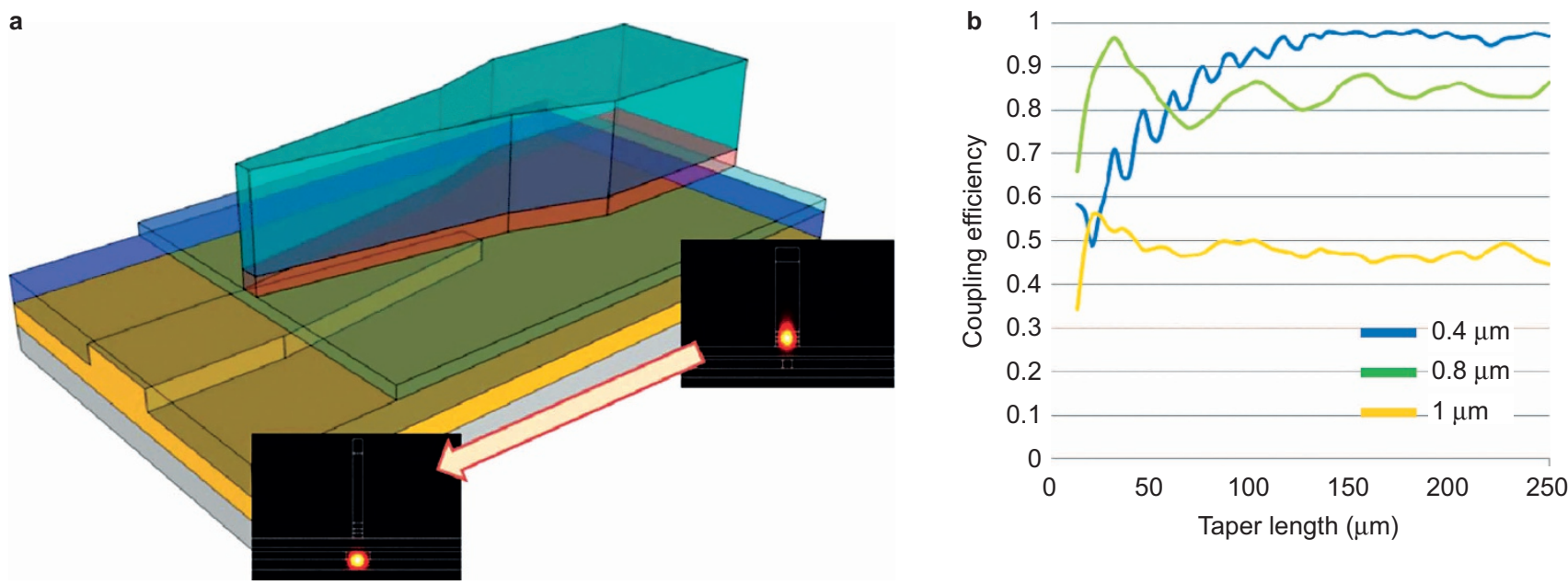

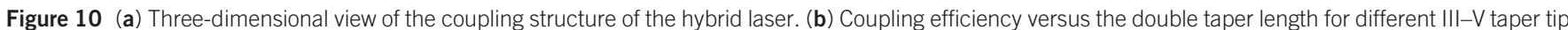
widths. Figures are taken from Ref. 117, (c) 2011 IEEE. 
a

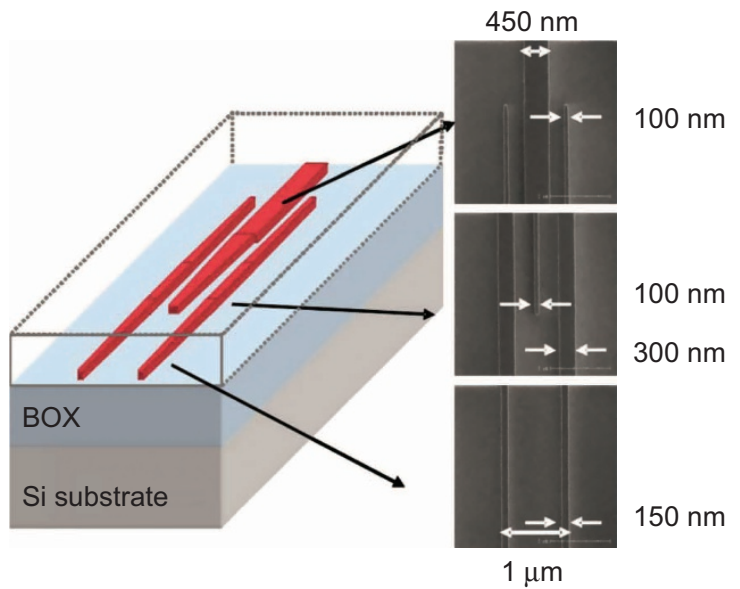

b

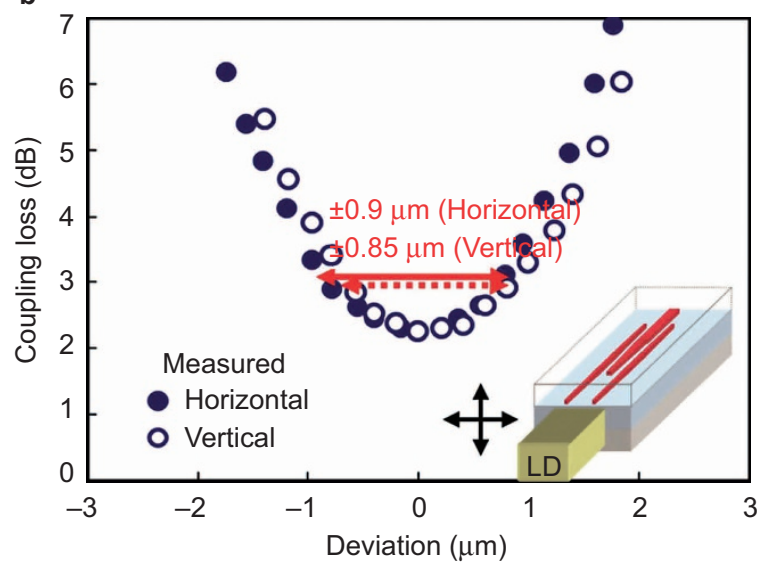

Figure 11 (a) Schematic structure and SEM images of the trident SSC. (b) Coupling losses between the LD and the trident SSC with various alignment deviations in the horizontal and vertical directions. Figures are taken from Ref. 119, (c) 2014 Chinese Laser Press.

Table 3 Key performance comparison of III-V-based Si laser integration approaches

\begin{tabular}{|c|c|c|c|c|c|c|c|}
\hline Integration approaches & $\lambda(\mu \mathrm{m})$ & Operation condition & $I_{\text {th }}(m A)$ & $P_{\text {out }}(\mathrm{mW})$ & WPE (\%) & $T_{\max }\left({ }^{\circ} \mathrm{C}\right)$ & Refs. \\
\hline Direct mounting integration & 1.53 & CW@25 & 12 & 39 & $\sim 20$ & NA & 6,118 \\
\hline \multirow{2}{*}{ Wafer bonding-based heterogeneous integration } & 1.55 & CW@20 & 9.4 & 11 & 7.6 & $>60$ & 120 \\
\hline & 1.36 & CW@20 & 18 & 35 & 14 & $>80$ & 121 \\
\hline
\end{tabular}

a $3 \mathrm{~dB}$ coupling loss between QD laser and silicon waveguide is assumed.

BCB bonding, have been successfully used in realizing III-V-based hybrid Si lasers integrated with other optical functional devices such as gratings, modulators, and detectors. Therefore, these lasers represent the best performance currently. Nevertheless, the bonding layers, such as BCB in BCB bonding and Si dioxide in direct bonding, usually produce a high thermal resistance. Therefore, heat dissipation should be considered to avoid the degradation of laser performance. QDs offer a possible solution for the direct hetero-epitaxial growth of III-V materials on Si because of its better tolerance to defects and the capability of filtering TDs. High-performance electrically pumped 1.3- $\mu \mathrm{m}$ InAs/GaAs QD lasers monolithically grown on Si have been successfully demonstrated. Furthermore, III-V material grown on the ELOG InP layer on Si realized comparable light emission intensities to those grown on InP substrate, indicating the availability of $1.55 \mu \mathrm{m}$ III-V laser monolithically grown on Si and the feasibility of direct hetero-epitaxial growth approaches for achieving monolithic integrated on-chip lasers.

\section{DISCUSSION AND CONCLUSIONS}

In this paper, the main approaches to achieving electrically pumped Si-based on-chip light sources have been reviewed in terms of the operating wavelength, pump condition, power consumption, fabrication, and thermal stability. Therefore, requirements must be considered when evaluating the performance of each candidate. Currently, no electrically pumped Er-related laser has been experimentally demonstrated because of the low power efficiency. The inherent limitation lies in the lower efficiency of the EL mechanism because it requires an extra energy transfer process to excite Er ions, rendering additional problems such as energy back-transfer in Er-doped siliconrich materials and the potential device instability in Er compound materials. Although Er-related light sources could work at HT with stable peak wavelengths because of an atomic luminescent mechanism, this source suffers from temperature quenching or high applied voltages, restricting the practicable application for on-chip integration.

For the Ge-on-Si laser, the birth of the first electrically pumped Ge laser and the recently demonstrated optically pumped GeSn laser are substantial steps to fill the gap in optoelectronic integration systems in spite of their imperfect performance. Benefiting from the large gain spectrum and ability to work at relative HT, the Ge laser is highly attractive in WDM systems and high-density OEIC. Moreover, the versatility of Ge opens the possibility of accomplishing photon emission, modulation, and detection simultaneously and establishes a monolithic integrated Ge-based photonic platform within a CMOS process flow, which may greatly reduce the process complexity and cost. However, a few critical barriers remain. The reduction of the threshold current for Ge lasers is certainly one of the biggest challenges, considering that no agreement is currently available on the underlying cause. Another substantial challenge is the serious redshift of the peak wavelength caused by the tensile strain and GeSn alloying. The peak wavelength would shift to the mid infrared region when $\mathrm{Ge}$ becomes a direct bandgap material, causing the Ge laser to be impractical in the available passive and active functional devices in silicon photonics because of the large operational wavelength mismatch and inability to achieve monolithic integration. In conclusion, Ge will play an increasing role in large-scale dense photonic integration because of the material compatibility and good performance at HT of the materials and because of overcoming processing issues involved in improving the high threshold currents. One of the main challenges is how to establish a trade-off between $n$-type doping, tens- 
ile strain, and GeSn alloying in terms of optimizing the performance of the Ge laser whereas also restricting the operating wavelength redshift.

III-V-based hybrid Si lasers produced via bonding techniques currently represent the best performance and display the highest potential for industrial use in the near future as an on-chip laser for silicon photonics. However, bonding techniques usually lead to poor heat dissipation because of the high thermal resistance of the bonding layer, thus severely degrading the laser performance at HT. This method is not a monolithic integration approach and has limitations for mass production. Therefore, III-V-based hybrid Si lasers produced via bonding techniques may not be a suitable solution for large-scale dense monolithic integration in terms of the yield and cost over the long term. In comparison, high-quality QD materials have been successfully grown on Si via direct hetero-epitaxial growth. Electrically pumped 1.3 $\mu \mathrm{m}$ QD lasers were demonstrated and could maintain lasing operation at up to $119{ }^{\circ} \mathrm{C}$ because of the delta-function-like density of states. Therefore, in the long run, III-V QD lasers, monolithically grown on $\mathrm{Si}$, could be more promising as on-chip lasers and may satisfy the requirements for low-cost, high-yield, temperatureinsensitive, and large-scale high-density monolithic integration.

\section{ACKNOWLEDGEMENTS}

This work was partially supported by the Major International Cooperation and Exchange Program of the National Natural Science Foundation of China under Grant 61120106012 and the Peking University 985 Startup Fund.

1 Cisco Visual Networking Index: Forecast and Methodology, 2013-2018. http://www. anatel.org.mx/docs/interes/Cisco_VNI_Forecast_and_Methodology.pdf (accessed 22 Dec 2014).

2 Rickman A. The commercialization of silicon photonics. Nat Photonics 2014; 8: 579 582.

3 Dai D, Bauters J, Bowers JE. Passive technologies for future large-scale photonic integrated circuits on silicon: polarization handling, light non-reciprocity and loss reduction. Light Sci App/2012; 1: e1; doi:10.1038/lsa.2012.1.

4 Thomson DJ, Gardes FY, Fedeli J, Zlatanovic S, Hu Y et al. 50-Gb/s silicon optical modulator. IEEE Photonic Tech Lett 2012; 24: 234-236.

5 Vivien L, Polzer A, Marris-Morini D, Osmond J, Hartmann JM et al. Zero-bias 40Gbit/s germanium waveguide photodetector on silicon. Opt Express 2012; 20: 1096-1101.

6 Urino Y, Usuki T, Fujikata J, Ishizaka M, Yamada K et al. High-density optica interconnects by using silicon photonics. In: Srivastava AK, editor. SPIE OPTO. International Society for Optics and Photonics; 2014. p901006.

7 Chaisakul P, Marris-Morini D, Frigerio J, Chrastina D, Rouifed MS et al. Integrated germanium optical interconnects on silicon substrates. Nat Photonics 2014; 8: 482488.

8 Heck MJR, Bowers JE. Energy efficient and energy proportional optical interconnects for multi-core processors: driving the need for on-chip sources. IEEE J Sel Top Quant 2014; 20: 8201012 .

9 Liang D, Bowers JE. Recent progress in lasers on silicon. Nat Photonics 2010; 4: 511 517.

10 Koshida N, Koyama H. Visible electroluminescence from porous silicon. Appl Phys Lett 1992; 60: 347-349.

11 Pavesi L, Dal Negro L, Mazzoleni C, Franzò G, Priolo F. Optical gain in silicon nanocrystals. Nature 2000; 408: 440-444.

12 Rong H, Liu A, Jones R, Cohen O, Hak D et al. An all-silicon Raman laser. Nature 2005; 433: 292-294.

13 Kenyon AJ. Erbium in silicon. Semicond Sci Tech 2005; 20: R65-R84.

14 Franzò G, Boninelli S, Pacifici D, Priolo F, lacona F et al. Sensitizing properties of amorphous $\mathrm{Si}$ clusters on the 1.54- $\mu \mathrm{m}$ luminescence of Er in Si-rich $\mathrm{SiO}_{2}$. Appl Phys Lett 2003; 82: 3871-3873.

15 Yerci S, Li R, Dal Negro L. Electroluminescence from Er-doped Si-rich silicon nitride light emitting diodes. Appl Phys Lett 2010; 97: 81109.

16 Miritello M, Lo Savio R, lacona F, Franzò G, Irrera A et al. Efficient luminescence and energy transfer in erbium silicate thin films. Adv Mater 2007; 19: 1582-1588.

17 Liu J, Sun X, Pan D, Wang X, Kimerling LC et al. Tensile-strained, n-type Ge as a gain medium for monolithic laser integration on Si. Opt Express 2007; 15: 11272 11277.

18 Liu J, Sun X, Camacho-Aguilera R, Kimerling LC, Michel J. Ge-on-Si laser operating at room temperature. Opt Lett 2010; 35: 679-681.

19 Camacho-Aguilera RE, Cai Y, Patel N, Bessette JT, Romagnoli M et al. An electrically pumped germanium laser. Opt Express 2012; 20: 11316-11320.
$20 \mathrm{He} \mathrm{G}$, Atwater HA. Interband transitions in $\mathrm{Sn}_{x} \mathrm{Ge}_{1-x}$ Alloys. Phys Rev Lett 1997; 79: 1937-1940.

21 Park H, Fang AW, Kodama S, Bowers JE. Hybrid silicon evanescent laser fabricated with a silicon waveguide and III-V offset quantum wells. Opt Express 2005; 13: 94609464

22 Fang AW, Park H, Cohen O, Jones R, Paniccia MJ et al. Electrically pumped hybrid AIGalnAs-silicon evanescent laser. Opt Express 2006; 14: 9203-9210.

23 Sun X, Zadok A, Shearn MJ, Diest KA, Ghaffari A et al. Electrically pumped hybrid evanescent Si/InGaAsP lasers. Opt Lett 2009; 34: 1345-1347.

24 Tanabe K, Watanabe K, Arakawa Y. III-V/Si hybrid photonic devices by direct fusion bonding. Sci Rep 2012; 2: 349.

25 Yang L, Armani DK, Vahala KJ. Fiber-coupled erbium microlasers on a chip. Appl Phys Lett 2003; 83: 825-826.

26 Polman A, Min B, Kalkman J, Kippenberg TJ, Vahala KJ. Ultralow-threshold erbiumimplanted toroidal microlaser on silicon. Appl Phys Lett 2004; 84: 1037-1039.

27 Jambois O, Berencen Y, Hijazi K, Wojdak M, Kenyon AJ et al. Current transport and electroluminescence mechanisms in thin $\mathrm{SiO}_{2}$ films containing $\mathrm{Si}$ nanoclustersensitized erbium ions. J Appl Phys 2009; 106: 063526.

28 Miller GM, Briggs RM, Atwater HA. Achieving optical gain in waveguide-confined nanocluster-sensitized erbium by pulsed excitation. J Appl Phys 2010; 108: 063109.

29 Dal Negro L, Yi JH, Michel J, Kimerling LC, Chang TWF et al. Light emission efficiency and dynamics in silicon-rich silicon nitride films. Appl Phys Lett 2006; 88: 233109.

30 Kazaks PA, Ganas PS, Green AES. Electron-impact excitation and ionization of atomic oxygen. Phys Rev A 1972; 6: 2169-2180.

31 Wang XJ, Nakajima T, Isshiki H, Kimura T. Fabrication and characterization of Er silicates on $\mathrm{SiO}_{2} / \mathrm{Si}$ substrates. App/ Phys Lett 2009; 95: 041906.

32 Pan A, Yin L, Liu Z, Sun M, Liu R et al. Single-crystal erbium chloride silicate nanowires as a Si-compatible light emission material in communication wavelength. Opt Mater Express 2011; 1: 1202-1209.

33 Jambois O, Ramírez JM, Berencén Y, Navarro-Urrios D, Anopchenko A et al. Effect of the annealing treatments on the electroluminescence efficiency of $\mathrm{SiO}_{2}$ layers doped with Si and Er. J Phys D: App/ Phys 2012; 45: 045103.

34 Ramírez JM, Jambois O, Berencén Y, Navarro-Urrios D, Anopchenko A et al. Polarization strategies to improve the emission of Si-based light sources emitting at 1.55 4 m. Mater Sci Eng: B 2012; 177: 734-738.

35 Krzyżanowska H, Ni KS, Fu Y, Fauchet PM. Electroluminescence from Er-doped $\mathrm{SiO}_{2} / \mathrm{nc}$-Si multilayers under lateral carrier injection. Mater Sci Eng: B 2012; 177: 1547-1550.

36 Fu Y. Light Emission and Slot Waveguide Effect in Er-doped $\mathrm{SiO}_{2} / \mathrm{Si}$ nanocrystalline Multilayer Structures[D]. New York: University of Rochester; 2012.

37 Li D, Wang F, Ren C, Yang D. Improved electroluminescence from silicon nitride light emitting devices by localized surface plasmons. Opt Mater Express 2012; 2: 872877.

38 Wang F, Li D, Yang D, Que D. Enhancement of light-extraction efficiency of $\mathrm{SiN}_{\mathrm{x}}$ light emitting devices through a rough Ag island film. Appl Phys Lett 2012; 100: 031113.

39 Prtljaga N, Navarro-Urrios D, Tengattini A, Anopchenko A, Ramírez JM et al. Limit to the erbium ions emission in silicon-rich oxide films by erbium ion clustering. Opt Mater Express 2012; 2: 1278-1285.

40 Navarro-Urrios D, Ferrarese Lupi F, Prtljaga N, Pitanti A, Jambois 0 et al. Copropagating pump and probe experiments on $\mathrm{Si}-\mathrm{nc}$ in $\mathrm{SiO}_{2}$ rib waveguides doped with Er: the optical role of non-emitting ions. App/ Phys Lett 2011; 99: 231114.

41 Jambois O, Gourbilleau F, Kenyon AJ, Montserrat J, Rizk R et al. Towards population inversion of electrically pumped $\mathrm{Er}$ ions sensitized by Si nanoclusters. Opt Express 2010; 18: 2230-2235.

42 Lo Savio R, Miritello M, Piro AM, Priolo F, lacona F. The influence of stoichiometry on the structural stability and on the optical emission of erbium silicate thin films. Appl Phys Lett 2008; 93: 021919.

43 Suh K, Lee M, Chang JS, Lee H, Park N et al. Cooperative upconversion and optical gain in ion-beam sputter-deposited $\mathrm{Er}_{x} \mathrm{Y}_{2-x} \mathrm{SiO}_{5}$ waveguides. Opt Express 2010; 18 : 7724-7731.

44 Vanhoutte M, Wang B, Zhou Z, Michel J, Kimerling LC. Direct demonstration of sensitization at $980 \mathrm{~nm}$ optical excitation in erbium-ytterbium silicates. In: 7th IEEE International Conference on Group IV Photonics. IEEE, 2010, pp 308-310.

45 Wang XJ, Wang B, Wang L, Guo RM, Isshiki $\mathrm{H}$ et al. Extraordinary infrared photoluminescence efficiency of $\mathrm{Er}_{0.1} \mathrm{Yb}_{1.9} \mathrm{SiO}_{5}$ films on $\mathrm{SiO}_{2} / \mathrm{Si}$ substrates. Appl Phys Lett 2011; 98: 071903.

46 Guo R, Wang B, Wang X, Wang L, Jiang L et al. Optical amplification in Er/Yb silicate slot waveguide. Opt Lett 2012; 37: 1427-1429.

47 Isshiki H, Jing F, Sato T, Nakajima T, Kimura T. Rare earth silicates as gain media for silicon photonics [Invited]. Photon Res 2014; 2: A45-A55.

48 Wang B, Guo R, Wang X, Wang L, Yin B et al. Large electroluminescence excitation cross section and strong potential gain of erbium in ErYb silicate. J Appl Phys 2013; 113: 103108

49 Yin L, Ning H, Turkdogan S, Liu Z, Nichols PL et al. Long lifetime, high density singlecrystal erbium compound nanowires as a high optical gain material. Appl Phys Lett 2012; 100: 241905.

50 Liu Z, Zhao G, Yin L, Ning CZ. Demonstration of net gain in an erbium chloride silicate single nanowire waveguide. In: CLEO: 2014. OSA: Washington, DC, 2014, pSM4H.4.

51 Yin L, Shelhammer D, Zhao G, Liu Z, Ning CZ. Erbium concentration control and optimization in erbium yttrium chloride silicate single crystal nanowires as a high gain material. Appl Phys Lett 2013; 103: 121902.

52 Liu J, Camacho-Aguilera R, Bessette JT, Sun X, Wang X et al. Ge-on-Si optoelectronics. Thin Solid Films 2012; 520: 3354-3360. 
53 Spitzer WG, Trumbore FA, Logan RA. Properties of heavily doped n-Type Germanium. J Appl Phys 1961; 32: 1822-1830.

54 van de Walle CG. Band lineups and deformation potentials in the model-solid theory. Phys Rev B 1989; 39: 1871-1883.

55 Ishikawa Y, Wada K, Cannon DD, Liu J, Luan $\mathrm{H}$ et al. Strain-induced band gap shrinkage in Ge grown on Si substrate. Appl Phys Lett 2003; 82: 2044-2046.

56 Sun XC, Liu JF, Kimerling LC, Michel J. Toward a germanium laser for integrated silicon photonics. IEEE J Sel Top Quant 2010; 16: 124-131.

57 Kim J, Bedell SW, Maurer SL, Loesing R, Sadana DK. Activation of implanted n-type dopants in Ge over the active concentration of $1 \times 10^{20} \mathrm{~cm}^{-3}$ using coimplantation of Sb and P. Electrochem Solid-St Lett 2010; 13: H12-H15.

58 Yu HY, Cheng SL, Griffin PB, Nishi Y, Saraswat KC. Germanium in situ doped epitaxial growth on Si for high-performance $\mathrm{n}^{+} / \mathrm{p}$-junction diode. IEEE Electr Device Lett2009; 30: 1002-1004

59 Sun X. Ge-on-Si light-emitting materials and devices for silicon photonics[D]. Massachusetts Institute of Technology; 2009

60 Cai Y, Camacho-Aguilera R, Bessette JT, Kimerling LC, Michel J. High phosphorous doped germanium: dopant diffusion and modeling. J App/ Phys 2012; 112: 034509.

61 Camacho-Aguilera RE, Cai Y, Bessette JT, Kimerling LC, Michel J. High active carrier concentration in n-type, thin film Ge using delta-doping. Opt Mater Express 2012; 2: 1462-1469.

62 Liu J, Cannon DD, Wada K, Ishikawa Y, Jongthammanurak S et al. Tensile strained Ge $\mathrm{p}$-i-n photodetectors on Si platform for $\mathrm{C}$ and $\mathrm{L}$ band telecommunications. App/ Phys Lett 2005; 87: 011110.

63 Liu J, Cannon DD, Wada K, Ishikawa Y, Jongthammanurak S et al. Silicidationinduced band gap shrinkage in Ge epitaxial films on Si. Appl Phys Lett 2004; 84: 660-662.

64 Huo Y, Lin H, Chen R, Rong Y, Kamins TI et al. MBE growth of tensile-strained Ge quantum wells and quantum dots. Front Optoelectron 2012; 5: 112-116.

65 Ghrib A, de Kersauson M, El Kurdi M, Jakomin R, Beaudoin G et al. Control of tensile strain in germanium waveguides through silicon nitride layers. Appl Phys Lett 2012; 100: 201104

66 Capellini G, Kozlowski G, Yamamoto Y, Lisker M, Wenger C et al. Strain analysis in SiN/ Ge microstructures obtained via Si-complementary metal oxide semiconductor compatible approach. J App/ Phys 2013; 113: 013513.

67 Jain JR, Hryciw A, Baer TM, Miller DAB, Brongersma ML et al. A micromachiningbased technology for enhancing germanium light emission via tensile strain. Nat Photonics 2012; 6: 398-405.

68 Trumbore FA. Solid solubilities and electrical properties of tin in germanium single crystals. J Electrochem Soc 1956; 103: 597-600.

69 Dutt B, Hai L, Sukhdeo DS, Vulovic BM, Gupta S et al. Theoretical analysis of GeSn alloys as a gain medium for a Si-Compatible laser. IEEE J Sel Top Quant 2013; 19 : 1502706.

70 Kouvetakis J, Menendez J, Chizmeshya AVG. Tin-based group IV semiconductors: new platforms for opto-and microelectronics on silicon. Annu Rev Mater Res 2006; 36: 497-554.

71 Lin H, Chen R, Huo Y, Kamins TI, Harris JS. Low-temperature growth of $\mathrm{Ge}_{1-x} \mathrm{Sn}_{\mathrm{x}}$ thin films with strain control by molecular beam epitaxy. Thin Solid Films 2012; 520: 3927-3930.

72 Sun G, Soref RA, Cheng HH. Design of an electrically pumped SiGeSn/GeSn/SiGeSn double-heterostructure midinfrared laser. J Appl Phys 2010; 108: 033107.

73 Chen R, Lin H, Huo Y, Hitzman C, Kamins TI et al. Increased photoluminescence of strain-reduced, high-Sn composition $\mathrm{Ge}_{1-x} \mathrm{Sn}_{\mathrm{x}}$ alloys grown by molecular beam epitaxy. App/ Phys Lett 2011; 99: 181125.

74 Sun X, Liu J, Kimerling LC, Michel J. Direct gap photoluminescence of $n$-type tensilestrained Ge-on-Si. Appl Phys Lett 2009; 95: 011911.

75 Sun X, Liu J, Kimerling LC, Michel J. Room-temperature direct bandgap electroluminesence from Ge-on-Si light-emitting diodes. Opt Lett 2009; 34: 11981200.

76 Cai H, Han Z, Wang X, Camacho-Aguilera RE. Analysis of threshold current behavior for bulk and quantum-well germanium laser structures. IEEE J Sel Top Quant 2013; 19: 1901009 .

77 Camacho-Aguilera R, Han Z, Cai Y, Kimerling LC, Michel J. Direct band gap narrowing in highly doped Ge. Appl Phys Lett 2013; 102: 152106.

78 Oehme M, Gollhofer M, Widmann D, Schmid M, Kaschel M et al. Direct bandgap narrowing in Ge LED's on Si substrates. Opt Express 2013; 21: 2206-2211.

79 Dutt B, Sukhdeo DS, Nam D, Vulovic BM, Saraswat KC. Roadmap to an efficient germanium-on-silicon laser: strain vs. n-Type doping. IEEE Photon J 2012; 4: 2002-2009.

80 Velha P, Dumas DC, Gallacher K, Millar R, Myronov M et al. Strained germanium nanostructures on silicon emitting at $>2.2 \mu \mathrm{m}$ wavelength. In: 10th International Conference on Group IV Photonics. IEEE, 2013, pp 142-143.

81 Süess MJ, Geiger R, Minamisawa RA, Schiefler G, Frigerio J et al. Analysis of enhanced light emission from highly strained germanium microbridges. Nat Photonics 2013; 7: 466-472.

82 Geiger R, Suess MJ, Minamisawa RA, Bonzon C, Schiefler G et al. Enhanced light emission from Ge micro bridges uniaxially strained beyond 3\%. In: 10th International Conference on Group IV Photonics. IEEE, 2013, pp 93-94.

83 Sukhdeo DS, Nam D, Kang J-H, Petykiewicz J, Lee JH et al. Direct bandgap germanium nanowires inferred from 5.0\% uniaxial tensile strain. In: 10th International Conference on Group IV Photonics. IEEE, 2013, pp 73-74.
84 Sukhdeo DS, Lin H, Nam D, Yuan Z, Vulovic BM et al. Approaches for a viable germanium laser: tensile strain, GeSn alloys, and n-type doping. In: 2013 Optica Interconnects Conference. IEEE, 2013, pp 112-113.

85 Wirths S, Geiger R, von den Driesch N, Mussler G, Stoica T et al. Lasing in directbandgap GeSn alloy grown on Si. Nat Photonics 2015; 9: 88-92.

86 Gerthsen D, Biegelsen DK, Ponce FA, Tramontana JC. Misfit dislocations in GaAs heteroepitaxy on (001) Si. J Cryst Growth 1990; 106: 157-165.

87 Mitze T, Schnarrenberger M, Zimmermann L, Bruns J, Fidorra F et al. Hybrid integration of III/V lasers on a silicon-on-insulator (SOI) optical board. In: IEEE International Conference on Group IV Photonics, 2005. IEEE, 2005, pp 210-212.

88 Hong T, Ran G, Chen T, Pan J, Chen W et al. A selective-area metal bonding InGaAsP-Si laser. IEEE Photonic Tech Lett 2010; 22: 1141-1143.

89 Hong T, Li Y, Chen W, Ran G, Qin G et al. Bonding InGaAsP/ITO/Si hybrid laser with ITO as cathode and light-coupling material. IEEE Photonic Tech Lett 2012; 24: 712 714.

90 Niklaus F, Enoksson P, Kälvesten E, Stemme G. Low-temperature full wafer adhesive bonding. J Micromech Microeng 2001; 11: 100-107.

91 van Campenhout J, Rojo-Romeo P, Van Thourhout D, Seassal C, Regreny P et al. Thermal characterization of electrically injected thin-film InGaAsP microdisk lasers on Si. J Lightwave Technol 2007; 25: 1543-1548.

92 Keyvaninia S, Muneeb M, Stanković S, van Veldhoven PJ, van Thourhout D et al. Ultra-thin DVS-BCB adhesive bonding of III-V wafers, dies and multiple dies to a patterned silicon-on-insulator substrate. Opt Mater Express 2012; 3: 35-46.

93 Esser RH, Hobart KD, Kub FJ. Improved low-temperature Si-Si hydrophilic wafer bonding. J Electrochem Soc 2003; 150: G228-G231.

94 Morizane K. Antiphase domain structures in GaP and GaAs epitaxial layers grown on $\mathrm{Si}$ and Ge. J Cryst Growth 1977; 38: 249-254.

95 Fischer R, Neuman D, Zabel H, Morkoc $\mathrm{H}$, Choi $\mathrm{C}$ et al. Dislocation reduction in epitaxial GaAs on Si(100). Appl Phys Lett 1986; 48: 1223-1225.

96 Groenert ME, Leitz CW, Pitera AJ, Yang V, Lee H et al. Monolithic integration of roomtemperature $\mathrm{cw}$ GaAs/AIGaAs lasers on Si substrates via relaxed graded GeSi buffer layers. J Appl Phys 2003; 93: 362-367.

97 Chriqui Y, Largeau L, Patriarche G, Saint-Girons G, Bouchoule S et al. Direct growth of GaAs-based structures on exactly (001)-oriented Ge/Si virtual substrates: reduction of the structural defect density and observation of electroluminescence at room temperature under CW electrical injection. J Cryst Growth 2004; 265: 53-59.

98 Cerutti L, Rodriguez JB, Tournie E. GaSb-based laser, monolithically grown on silicon substrate, emitting at $1.55 \mathrm{~m}$ at room temperature. IEEE Photonic Tech Lett 2010; 22: $553-555$

99 Olsson F, Xie M, Lourdudoss S, Prieto I, Postigo PA. Epitaxial lateral overgrowth of InP on Si from nano-openings: theoretical and experimental indication for defect filtering throughout the grown layer. J Appl Phys 2008; 104: 093112.

100 Langdo TA, Leitz CW, Currie MT, Fitzgerald EA, Lochtefeld A et al. High quality Ge on Si by epitaxial necking. Appl Phys Lett 2000; 76: 3700-3702.

101 Wang Z, Junesand C, Metaferia W, Hu C, Wosinski L et al. III-Vs on Si for photonic applications-A monolithic approach. Mater Sci Eng: B 2012; 177: 1551-1557.

102 Kataria H, Metaferia W, Junesand C, Zhang C, Julian N et al. Simple epitaxial latera overgrowth process as a strategy for photonic integration on silicon. IEEE J Sel Top Quant 2014; 20: 8201407.

103 Balakrishnan G, Huang S, Khoshakhlagh A, Dawson LR, Xin YC et al. High quality AISb bulk material on Si substrates using a monolithic self-assembled quantum dot nucleation layer. J Vac Sci Technol B: Microelectron Nanometer Struct 2005; 23 1010-1012.

104 Mi Z, Bhattacharya P, Yang J, Pipe KP. Room-temperature self-organised $\mathrm{n}_{0.5} \mathrm{Ga}_{0.5} \mathrm{As}$ quantum dot laser on silicon. Electron Lett 2005; 41: 742-744.

105 Liu H, Wang T, Jiang Q, Hogg R, Tutu F et al. Long-wavelength InAs/GaAs quantum-do laser diode monolithically grown on Ge substrate. Nat Photonics 2011; 5: 416-419.

106 Lee A, Jiang Q, Tang M, Seeds A, Liu H. Continuous-wave InAs/GaAs quantum-dot laser diodes monolithically grown on Si substrate with low threshold current densities. Opt Express 2012; 20: 22181-22187.

107 Wang T, Liu H, Lee A, Pozzi F, Seeds A. 1.3- $\mu \mathrm{m}$ InAs/GaAs quantum-dot lasers monolithically grown on Si substrates. Opt Express 2011; 19: 11381-11386.

108 Lee AD, Jiang Q, Tang M, Zhang Y, Seeds AJ. InAs/GaAs quantum-dot lasers monolithically grown on Si, Ge, and Ge-on-Si substrates. IEEE J Sel Top Quant 2013; 19 1901107.

109 Tang M, Chen S, Wu J, Jiang Q, Dorogan VG et al. 1.3- $\mu \mathrm{m}$ InAs/GaAs quantum-dot lasers monolithically grown on $\mathrm{Si}$ substrates using InAIAs/GaAs dislocation filter layers. Opt Express 2014; 22: 11528-11535.

110 Chen SM, Tang MC, Wu J, Jiang Q, Dorogan VG et al. $1.3 \mu \mathrm{m}$ InAs/GaAs quantum-dot laser monolithically grown on $\mathrm{Si}$ substrates operating over $100^{\circ} \mathrm{C}$. Electron Lett 2014 ; 50: 1467-1468.

111 Liu AY, Zhang C, Norman J, Snyder A, Lubyshev D et al. High performance continuous wave $1.3 \mu \mathrm{m}$ quantum dot lasers on silicon. Appl Phys Lett 2014; 104: 041104.

112 Fang AW, Koch BR, Jones R, Lively E, Liang D et al. A distributed Bragg reflector silicon evanescent laser. IEEE Photonic Tech Lett 2008; 20: 1667-1669.

113 Fang AW, Lively E, Kuo Y, Liang D, Bowers JE. A distributed feedback silicon evanescent laser. Opt Express 2008; 16: 4413-4419.

114 Liang D, Fiorentino M, Okumura T, Chang H, Spencer DT et al. Electrically-pumped compact hybrid silicon microring lasers for optical interconnects. Opt Express 2009, 17: 20355-20364

115 van Campenhout J, Rojo-Romeo P, Regreny P, Seassal C, Van Thourhout D et al. Electrically pumped InP-based microdisk lasers integrated with a nanophotonic silicon-on-insulator waveguide circuit. Opt Express 2007; 15: 6744-6749. 
116 Lamponi M, Keyvaninia S, Pommereau F, Brenot $\mathrm{R}$, de Valicourt $\mathrm{G}$ et al. Heterogeneously integrated InP/SOI laser using double tapered single-mode waveguides through adhesive die to wafer bonding. In: 7th IEEE International Conference on Group IV Photonics. IEEE, 2010, pp 22-24.

117 Lamponi M, Keyvaninia S, Jany C, Poingt F, Lelarge F et al. Low-threshold heterogeneously integrated InP/SOI lasers with a double adiabatic taper coupler. IEEE Photonic Tech Lett 2012; 24: 76-78.

118 Shimizu T, Hatori N, Okano M, Ishizaka M, Urino Y et al. High density hybrid integrated light source with a laser diode array on a silicon optical waveguide platform for interchip optical interconnection. In: 8th IEEE International Conference on Group IV Photonics. IEEE, 2011, pp 181-183.

119 Urino Y, Usuki T, Fujikata J, Ishizaka M, Yamada K et al. High-density and widebandwidth optical interconnects with silicon optical interposers [Invited]. Photon Res 2014; 2: A1-A7.

120 Tanaka S, Jeong S, Sekiguchi S, Kurahashi T, Tanaka Y et al. High-output-power, single-wavelength silicon hybrid laser using precise flip-chip bonding technology. Opt Express 2012; 20: 28057-28069.

121 Koch BR, Norberg EJ, Kim B, Hutchinson J, Shin J-H et al. Integrated silicon photonic laser sources for telecom and datacom. In: Optical Fiber Communication Conference/
National Fiber Optic Engineers Conference 2013. OSA: Washington, DC, 2013, pPDP5C.8.

122 Wang Y, Wei Y, Huang Y, Tu Y, Ng D et al. Silicon/III-V laser with super-compact diffraction grating for WDM applications in electronic-photonic integrated circuits. Opt Express 2011; 19: 2006-2013.

123 Intel Milestone Confirms Light Beams Can Replace Electronic Signals for Future Computers. http://www.intel.com/pressroom/archive/releases/2010/20100727comp_ sm.htm\#story (accessed 22 Dec 2014).

124 Jain SR, Sysak MN, Kurczveil G, Bowers JE. Integrated hybrid silicon DFB laser-EAM array using quantum well intermixing. Opt Express 2011; 19: 13692-13699.

(c) (i) $\odot$ This work is licensed under a Creative Commons Attribution-

ND NonCommercial-NoDerivs 4.0 Unported License. The images or other third party material in this article are included in the article's Creative Commons license, unless indicated otherwise in the credit line; if the material is not included under the Creative Commons license, users will need to obtain permission from the license holder to reproduce the material. To view a copy of this license, visit http://creativecommons.org/licenses/ by-nc-nd/4.0/ 